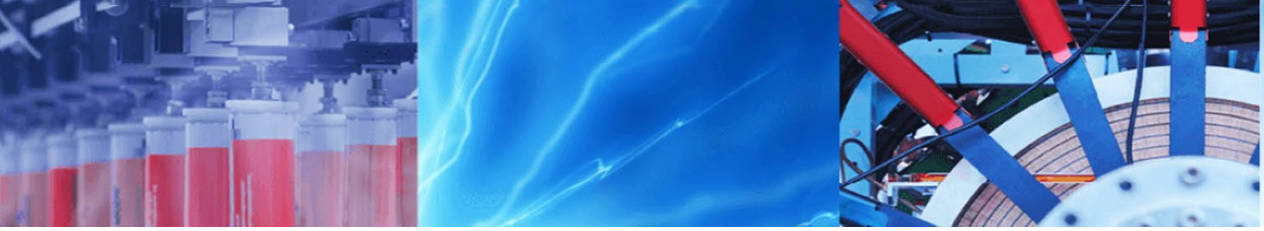

Research Article

\title{
On the stress-induced photon emission from organism: II, how will the stress-transfer kinetics affect the photo-genesis?
}

\author{
Daqing Piao' ${ }^{1}$
}

Received: 2 February 2020 / Accepted: 17 August 2020 / Published online: 25 August 2020

(c) Springer Nature Switzerland AG 2020

\begin{abstract}
Much remains to be identified for the temporal course of stress-induced photon emission (PE) following stress of various types including but not limited to light. Induced PE often decays hyperbolically; yet, it is not uncommon for induced PE to manifest decay patterns that are various combinations of first-order responses. Induced PE also presented transient patterns characteristic of second-order responses. A soliton-based photon-storage model addressed the hyperbolic decay pattern of induced PE; however, there are questions regarding non-hyperbolic decay as well as the large range of delayand-decay scales of induced PE. This work offers an alternative interpretation of the temporal course of induced PE when stressed upon an organism. It is proposed that the surface photon emission of induced PE due to a stress involves two causally sequential phases: a stress-transfer phase that transforms the stress to photo-genesis, and a photon-propagation phase that transmits the photons from the site of photo-genesis to surface emission. Part I has argued that a retarded or slow stress-transfer phase is necessary to explain induced PE occurring/lasting at a timescale several orders of magnitude later/longer than the photon propagation delay due to tissue scattering after stress-removal. Part II models the kinetics of the stress-transfer phase that sources the photo-genesis with a linear-system approach. The analysis illustrates how a single stress-transfer pathway may manifest various photo-genesis patterns in responding to the same stressinput, and why a single kinetic pattern of photo-genesis may arise from multiple paths of stress transfer. The theoretical insights may help devise stress-control strategies to enhance the yield of induced PE for more mechanistic discoveries and potentiating broader applications.
\end{abstract}

Keywords Ultraweak biophoton emission · Stress-induced photon emission · Decay kinetics · Stress transfer · Photogenesis

\section{Introduction}

Living organisms emit very weak light that differs from the bioluminescence produced by luciferin-luciferase systems [1]. This ultraweak photon emission (UPE) is sourced by the transition of excited biological molecules, mostly reactive oxygen species (ROS) to lower-energy states. ROS are generated in cell at a fixed rate by oxidation reduction reactions during normal cellular respiration, but are toxic to living cells due to impairing membrane functioning, reducing enzyme activity, and damaging DNA [1]. When in homeostasis, the organism employs a variety of mechanisms to scavenge the ROS to maintain the concentration of ROS at very low levels. As a result, the luminescence intensity of the baseline spontaneous UPE of a living healthy organism is extremely low. However, when living organisms become stressed, the concentration of ROS increases and strong induced PE is observed [2].

Induced $\mathrm{PE}$, or the increase in surface-emitted photons in excess of the baseline level of spontaneous UPE

Daqing Piao, daqing.piao@okstate.edu | 'School of Electrical and Computer Engineering, Oklahoma State University, Stillwater, OK 74078, USA. 
has been observed in association with an external stress of various types including but not limited to light $[3,4]$. The increase in photon count in induced PE over the baseline spontaneous UPE has been attributed to oxidative burst caused by stress-activated metabolic responses that perturbs the homeostasis via pathways that are still open to discussion [5-7]. Since induced PE responds to stress through metabolic pathway resulting in oxidative burst, modulating stress to modulate induced PE infers the oxidative stress pathway $[8,9]$. Identifying the specific route or mechanism of stress-action will rely upon determining how the induced PE responds to a stress that is controllable. In terms of devising time-resolved assessment of induced PE with the access to stress-control, four cases may be conceptually expected as illustrated in Fig. 1 concerning a stress that is simplified as a pulse. (A), the induced PE would respond instantaneously to a stress so the temporal profile of the induced PE would duplicate that of the stress. (B), the induced PE would appear instantaneously following a stress but a slower temporal change could cause a rising phase at the onset and a falling phase at the removal of the stress. (C), the induced PE would appear after the onset of a stress with a delay shorter than the duration of the stress. (D), the induced PE would appear after the removal of a stress. There are thus two aspects to determine for the temporal course of induced PE with respect to the temporal course of the stress: (1) a time delay or phase shift issue referring to the time it takes for the change of the photon count (over the baseline UPE) to occur with respect to the change of the stress; (2) the kinetic decay or dynamic change issue relevant to the time it takes for the change of photon count to stabilize after the change of stress sets. If the stress-removal is to be followed instantaneously by initiation of photon counting (a clean switching that is however impractical), any time lag between the stress-removal and the registration of the increase in photon counts over the baseline UPE would be the combined effects of the aforementioned delay and decay, both of which are associated with the pathway determining how the stress may activate photo-genesis in excess of the baseline level.

Of induced PE resulting from stress of various kinds, those responding to light or photic stress have registered the shortest device-limited time lag, a minimum of $8.5 \mu \mathrm{s}$ after the stress-removal [10]. And the intensity of the initial peak of induced PE in response to photic stress has shown to reach a level several orders of magnitude stronger than the baseline spontaneous UPE $[8,11]$. Stresses of other types, such as chemical [3], mechanical [12], thermal/environmental [1], radiative [13], electrical [14], and magnetic [15] have shown to result in induced PE of relatively smaller change over the baseline level than the photic stress. Non-photic stress also seems to cause induced PE to occur much later after the stressremoval and decay much slower than that due to photic stress [16]. Induced PE caused by photic stress generally decays hyperbolically $[17,18]$. The hyperbolic decay pattern of induced PE was projected to indicate coherence of the underlying photo-genesis $[3,19]$ of which the complete mechanism is still outstanding. It is worth noting that, there are many presentations of the kinetic decay of induced PE that do not fit to a hyperbolic pattern.

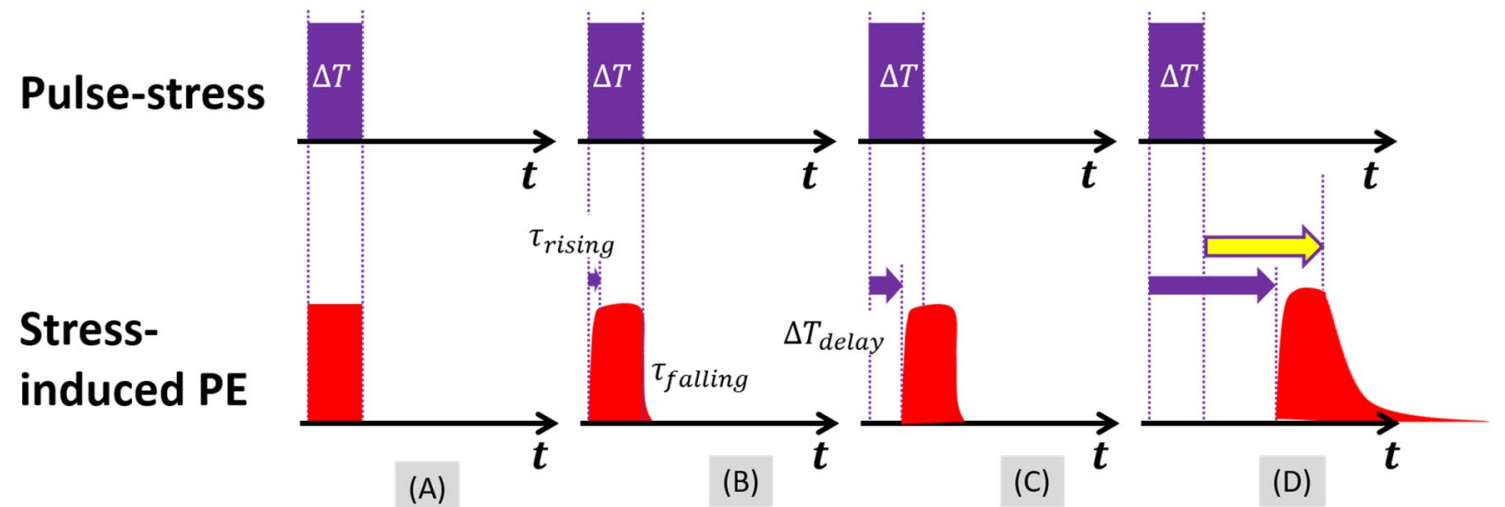

Fig. 1 The temporal profiles of induced PE and the stress that can be externally controlled may reveal the four cases as shown. a the induced PE responds instantaneously to a stress so the temporal profile of the induced PE duplicates that of the stress. $\mathbf{b}$ the induced PE appears instantaneously following a stress but there is a slower temporal change to cause a rising phase at the onset and a falling phase at the removal of the stress. $c$ the induced PE appears after the onset of a stress with a delay shorter than the duration of the stress. $\mathbf{d}$ the induced PE appears after the removal of a stress.
There are thus two aspects of the temporal course of induced PE with respect to the onset or removal of stress: (1) a time delay or phase shift issue referring to the time it takes for the change of the photon count to occur with respect to the change of the stress; (2) the kinetic decay or dynamic change issue relevant to the time it takes for the change of photon count to stabilize after the change of stress sets. Most experimental reports registered induced PE after the removal of stress so the induced PE first elevated from and then reduced to the baseline level of spontaneous UPE 
Induced PE revealing single-exponential decay patterns with long time constants was not uncommon to plants exposed to non-photic stresses [20-23]. Induced PE showing double-exponential decay patterns are also appreciable from organisms exposed to photic [24], mechanical $[12,21,25,26]$, chemical [16, 27-33], and radioactive [13] stresses. Besides the various decay patterns of induced $\mathrm{PE}$, the total duration of induced $P E$ varies over a large range after the stress-removal. Some phenomena of induced PE have the decay of photons registered continuously over several seconds to even hours after the stress-removal [16]. Not counting the device-specific delay between the removal of stress and the counting of induced $P E$, these decay patterns had been modeled with a coherent-state theory projecting correlated coherent many-solitons existing in organism $[18,34]$ as initially proposed by Popp $[19,35]$. The soliton-based models have been successful for interpreting the hyperbolic decay pattern of induced PE when stressed by ultraviolet light, and substantiating the nonlinear dependence of the yield of induced PE on the intensity of photic stress $[36,37]$. However, even induced PE responding to photic stress could present a decay pattern differing from the hyperbolic one, such as a multi-exponential decay pattern [11]. Other patterns of decay reported for induced PE include single exponential decay pattern [23], occasionally mixed with doubleexponential patterns [14], and transient patterns of rapid oscillation overlapping on a slowly changing global profile and biphasic patterns when responding to a temporally monotonic stress [30, 31, 38]. Those rapid oscillatory and biphasic patterns when responding to simple monotonic stress cannot be justified by first-order responses but are conveniently treated as second-order responses. Besides the varieties of the decay pattern of induced PE in responding to stress of various types, the broad scales of the duration of induced PE that could last hours to even days after the stress-removal is also not easily accountable by a soliton-based model addressing the hyperbolic decay pattern alone.

This work that contains two parts articulates a phenomenological kinetic-model framework for alternative accounting of the various temporal patterns of induced PE following bolus and step stresses that would form the basis for the analysis of the patterns caused by more complicated stress. Part I [39] has postulated that the temporal profiles of induced PE are governed by two causally sequential phases: a stress-transfer phase that transforms the external stress to photo-genesis, and a photon-propagation phase that transmits the photons sourced by photo-genesis to surface emission. Any delay between the change of stress and the change of surface photon-count must be no shorter than the cumulative time taken for the stress to activate photo-genesis and the light generated to make surface emission. Wherever the sites of photo-genesis are, the emitted photons in excess of the baseline spontaneous UPE will experience scattering and absorption before reaching the surface. Therefore, the photon-paths from the sites of photo-genesis to the surface site will be modulated by tissue scattering, which will cause temporal spread or broadening of the photon lifetime in tissue. Because induced PE concerns the health of organism including human that could be orders of magnitude larger in size comparing to photon scattering pathlength in organism, knowing how much temporal spread or broadening of the photon lifetime can present in a human-size organism indicates how faithful the temporal course of induced PE represents the stresstransfer process. Analysis of time-resolved photon diffusion in Part I for an extremely scattering tissue (a reduced scattering coefficient of $\left.500 \mathrm{~cm}^{-1}\right)$ over a spherical domain comparable to human ( $40 \mathrm{~cm}$ in diameter) reveals that the scattering-caused temporal spread (or lifetime-in-tissue) to the surface-emitted photons will not surpass $100 \mathrm{~ns}$. This suggests that, induced PE registered 100 ns later than the stress-removal or lasting much longer than the stress-duration involves a much retarded or slower phase of photo-genesis. The temporal characteristics of the much retarded or slower photo-genesis will then dictate the temporal profile of the surface emission observed as induced PE. As the temporal characteristics of photo-genesis sourcing surface photon emission will be governed by the stress-transfer phase, knowing how a specific stresstransfer kinetics may affect photo-genesis will be valuable to the mechanistic probing using induced PE by means of stress-control or stress-modulation.

This part II uses a linear-system approach to model the kinetic behavior expected for the stress-transfer pathway preceding the photo-genesis phase to project the photogenic responses to a bolus or a step stress for future analysis of photo-genic responses to stresses of arbitrary profiles. The stress-transfer phase is assumed to involve one of the following types of systems having constant kinetic rates: single first-order low-pass pathway, two or more inparallel first-order low-pass pathways, single second-order low-pass pathway with various damping factors, and single second-order band-pass pathway with various damping factors. A single first-order low-pass transfer pathway with a time-varying kinetic rate is also considered. The photo-genic responses of these model pathways to bolus and step inputs are compared numerically to illustrate how a single stress-transfer pathway may manifest various photo-genesis patterns in responding to the same stressinput, and why a single kinetic pattern of photo-genesis may arise from multiple paths of stress transfer. Unique patterns of stress-transfer responses are identified, and the 
potential of stress-modulation techniques for probing the stress-transfer pathways is discussed.

\section{Model basis}

Each photon of induced PE detected at the organism surface in response to a stress has to originate somewhere within the organism and traverse to the surface. A temporal spread of surface-emitted photons after stress-removal that occurs much later and lasts much longer than what can be caused by tissue-scattering can only be explained by a retarded or slower process of the photon-production in the absence of secondary photon production. We thus consider a sequential process giving rise to the surface emission of induced PE as suggested by Part I and conceptually illustrated in Fig. 2. The stress of various types is assumed to be applied at a spatial location of $\vec{\chi}^{\prime \prime}$ of the organism and an instant of $t^{\prime}$ as represented by $i_{\text {stress }}\left(\vec{\chi}^{\prime \prime}, t^{\prime}\right)$. The stress is considered to cause a lumped change to a chemical potential of metabolic aspect represented by $\odot_{1}\left(\vec{\chi}^{\prime}, t^{\prime}\right)$ at a kinetic rate of $K_{01}\left(\vec{\chi}^{\prime \prime} \Rightarrow \vec{\chi}^{\prime}\right)$ (1/second). The change of the $\odot_{1}\left(\vec{\chi}^{\prime}, t^{\prime}\right)$ is assumed to activate photo-genesis at the same spatial location of $\vec{\chi}^{\prime}$ with an activation kinetic rate of $K_{1 \mho}\left(\vec{\chi}^{\prime}\right)(1 /$ second), by triggering biological chain reactions affecting excited oxidative states of the organism. The photons then traverse to the surface position of $\vec{\chi}$ at a later time $t$ to be acquired by a photodetector as the induced PE.

In the case of much retarded or slower photon sourcing in comparison with the photon lifetime in tissue the temporal profile of the photons measured at the tissue surface shall be the convolution of the source temporal profile with the temporal point-spread function $\hat{\Psi}_{\mathrm{PSF}}\left(\vec{\chi}^{\prime}, 0 \mid \vec{\chi}, t\right)$ associated with the photon propagation process. If the source of temporal spread at $\vec{\chi}^{\prime}$ is spatially impulsive, as is represented by $\hat{q}_{\text {phot }}\left(\vec{\chi}^{\prime}, t^{\prime}\right)=\delta\left(\vec{\chi}^{\prime}\right) \mathfrak{q}\left(t^{\prime}\right), t^{\prime} \geq 0$, the convolution results in the actual "measured" photon count $\hat{\Psi}_{\text {meas }}\left(\vec{\chi}^{\prime}, t^{\prime} \mid \vec{\chi}, t\right)$ as the following:

$$
\hat{\Psi}_{\text {meas }}\left(\vec{\chi}^{\prime}, t^{\prime} \mid \vec{\chi}, t\right)=\hat{\Psi}_{\mathrm{PSF}}\left(\vec{\chi}^{\prime}, t^{\prime} \mid \vec{\chi}, t\right) \circledast \hat{q}_{\text {phot }}\left(\vec{\chi}^{\prime}, t\right)=\int_{-\infty}^{\infty} \hat{\Psi}_{\mathrm{PSF}}\left(\vec{\chi}^{\prime}, t^{\prime} \mid \vec{\chi}, \tau\right) \cdot \mathfrak{q}(t-\tau) \mathrm{d} \tau \text {. }
$$

$\hat{\Psi}_{\text {meas }}\left(\vec{\chi}^{\prime}, t^{\prime} \mid \vec{\chi}, t\right)=\hat{\Psi}_{\mathrm{PSF}}\left(\vec{\chi}^{\prime}, t^{\prime} \mid \vec{\chi}, t\right) \circledast \hat{q}_{\mathrm{phot}}\left(\vec{\chi}^{\prime}, t^{\prime}\right)=\int_{-\infty}^{\infty} \mathbb{Q}\left(\vec{\chi}^{\prime}-\vec{x}\right) \cdot\left[\int_{-\infty}^{\infty} \hat{\Psi}_{\mathrm{PSF}}\left(\vec{x}, t^{\prime} \mid \vec{\chi}, \tau\right) \cdot \mathbb{q}(t-\tau) \mathrm{d} \tau\right] \mathrm{d} \vec{x}$.

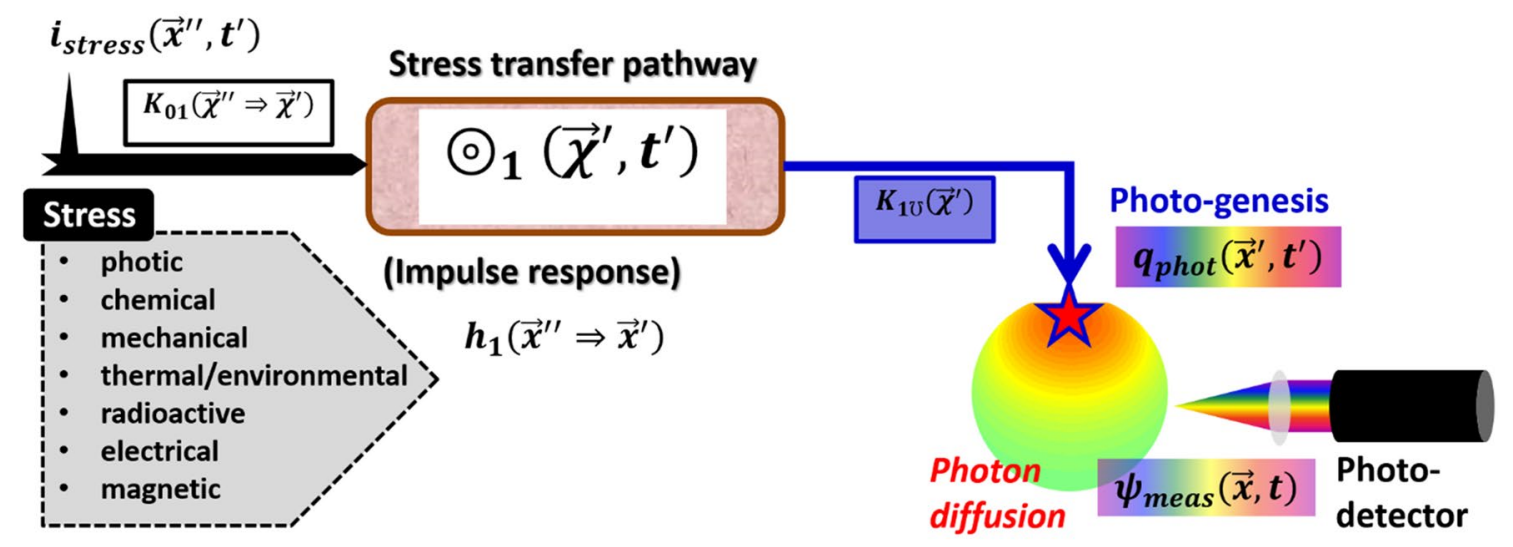

Fig. 2 The induced PE is assumed to involve a stress-transfer phase and a photon-propagation phase. Many types of stress may cause induced PE. The types of stress framed in the dashed-area include photic, chemical, mechanical, thermal/environmental, radioactive, electrical, and mechanical. The external stress is represented by
When the temporally spread source at $\vec{\chi}^{\prime}$ is also spatially spread as is represented by $\hat{q}_{\text {phot }}\left(\vec{\chi}^{\prime}, t^{\prime}\right)=\mathbb{Q}\left(\vec{\chi}^{\prime}\right) \mathbb{q}\left(t^{\prime}\right)$, $t^{\prime} \geq 0$, the spatial convolution will also contribute to the composite temporal profile of the surface-emitted photons as the following:

\section{SN Applied Sciences}

$i_{\text {stress }}\left(\vec{\chi}^{\prime \prime}, t^{\prime}\right)$. This stress is considered to cause a change to a potential of metabolic aspect represented by $\odot_{1}\left(\vec{\chi}^{\prime}, t^{\prime}\right)$, at a kinetic rate of $K_{01}\left(\vec{\chi}^{\prime \prime} \Rightarrow \vec{\chi}^{\prime}\right)(1 /$ second). The change of the metabolic potential $\odot_{1}\left(\vec{\chi}^{\prime}, t^{\prime}\right)$ is considered photo-genetic at a kinetic rate of $K_{1 \mho}\left(\vec{\chi}^{\prime}\right)$ $(1 /$ second) to produce the photons traversing to the surface 
Equation (2) will be relevant if the spatial extent of the entity that sources the induced PE is known. At present however the spatial extent of what sources induced PE is prohibiting to specify. This work thus has limited the discussion of photo-genesis that sources surface emission of induced PE to be spatially impulsive. For a temporal impulse response $\hat{\Psi}_{\mathrm{PSF}}\left(\vec{\chi}^{\prime}, 0 \mid \vec{\chi}, t\right)$ that is significantly faster than the temporal profile of the source generation $\hat{q}_{\text {phot }}\left(\vec{\chi}^{\prime}, t^{\prime}\right)=\delta\left(\vec{\chi}^{\prime}\right) \mathfrak{q}\left(t^{\prime}\right)$, the temporal impulse response $\hat{\Psi}_{\mathrm{PSF}}\left(\vec{\chi}^{\prime}, 0 \mid \vec{\chi}, t\right)$ can be approximated as a Dirac delta function for the convolution, so the temporal profile of the convolution of any function with a Dirac delta function will be dictated by the temporal profile of the host function. In referring to Part I, one can find that the temporal spread expected for photons diffusely propagating over a line-of-sight distance of $20 \mathrm{~cm}$ in a tissue of extremely high reduced scattering coefficient of $500 \mathrm{~cm}^{-1}$ with a detection dynamic range of 6 orders of magnitude is less than 80 ns. The shortest device-delay time of $8.5 \mu$ s currently registered for induced $\mathrm{PE}$ is $>100$ times longer than the $80 \mathrm{~ns}$ temporal spread that is practically the upper limit of the temporal scale of $\hat{\Psi}_{\mathrm{PSF}}\left(\vec{\chi}^{\prime}, 00 \mid \vec{\chi}, t\right)$. Therefore, the temporal profile of photon acquisition at delay times longer than $8.5 \mu \mathrm{s}$ will faithfully follow the temporal profile of the photon generation of $\hat{q}\left(\vec{\chi}^{\prime}, t\right)=q(t)$. For this reason, the analysis here is restricted to the stress-transfer phase, concerning how the kinetics of photo-genesis of $\hat{q}_{\text {phot }}\left(\vec{\chi}^{\prime}, t^{\prime}\right)=\delta\left(\vec{\chi}^{\prime}\right) \mathfrak{q}\left(t^{\prime}\right)$ may be affected by a few stresstransfer pathways that are modeled as linear time-invariant systems.

We now consider some practical or regular temporal patterns concerning how the stress may be applied. A single bolus stimulation with a stress intensity of $A_{\text {stress }}$ is expressed as:

$i_{\text {bolus }}\left(\vec{\chi}^{\prime \prime}, t^{\prime}\right)=A_{\text {stress }} \cdot \delta\left(\vec{\chi}-\vec{\chi}^{\prime \prime}\right) \cdot \delta\left(t^{\prime}\right)$.

A bolus stress of intensity $A_{\text {stress }}$ that is applied repetitively at an interval of $\mathrm{T}$ for a total of $\mathrm{N}$ times is represented by:

$$
\begin{aligned}
i_{\text {bolus }}^{\text {rep }}\left(\vec{\chi}^{\prime \prime}, t^{\prime}\right) & =A_{\text {stress }} \cdot \delta\left(\vec{\chi}-\vec{\chi}^{\prime \prime}\right) \cdot \sum_{n=0}^{N} \delta\left(t^{\prime}-n T\right) \\
& =\sum_{n=0}^{N} i_{\text {bolus }}\left[\vec{\chi}^{\prime \prime},\left(t^{\prime}-n T\right)\right] .
\end{aligned}
$$

A continuous stress with an intensity of $A_{\text {stress }}$ is characterized by

$i_{\text {step }}\left(\vec{\chi}^{\prime \prime}, t^{\prime}\right)=A_{\text {stress }} \cdot \delta\left(\vec{\chi}-\vec{\chi}^{\prime \prime}\right) \cdot u\left(t^{\prime}\right)$,

where $u\left(t^{\prime}\right)$ is the Heaviside function. A stress of $A_{\text {stress }}$ applied over a duration of $\Delta T$ is:

$$
\begin{aligned}
i_{\text {pulse }}\left(\vec{\chi}^{\prime \prime}, t^{\prime}\right) & =A_{\text {stress }} \cdot \delta\left(\vec{\chi}-\vec{\chi}^{\prime \prime}\right) \cdot\left[u\left(t^{\prime}\right)-u\left(t^{\prime}-\Delta T\right)\right] \\
& =i_{\text {step }}\left(\vec{\chi}^{\prime \prime}, t^{\prime}\right)-i_{\text {step }}\left[\vec{\chi}^{\prime \prime},\left(t^{\prime}-\Delta T\right)\right] .
\end{aligned}
$$

A stress with an intensity of $A_{\text {stress }}$ that is applied over a duration of $\Delta T$ and repeated with an interval of $T$ for a total of $\mathrm{N}$ times is expressed by:

$$
\begin{aligned}
i_{\text {pulse }}^{\text {rep }}\left(\vec{x}^{\prime \prime}, t^{\prime}\right)= & A_{\text {stress }} \cdot \delta\left(\vec{x}-\vec{x}^{\prime \prime}\right) \cdot \sum_{n=0}^{N}\left[u\left(t^{\prime}-n T\right)-u\left(t^{\prime}-\Delta T-n T\right)\right] \\
& =\sum_{n=0}^{N} i_{\text {pulse }}\left[\vec{x}^{\prime \prime},\left(t^{\prime}-n T\right)\right] \\
& =\sum_{n=0}^{N}\left\{i_{\text {step }}\left[\vec{x}^{\prime \prime},\left(t^{\prime}-n T\right)\right]-i_{\text {step }}\left[\vec{x}^{\prime \prime},\left(t^{\prime}-\Delta T-n T\right)\right]\right\} .
\end{aligned}
$$

The impulse response of a stress-transfer pathway that transfers the stress occurring at a spatial location of $\vec{\chi}^{\prime \prime}$ to the photo-genesis at a different location of $\vec{\chi}^{\prime}$ is denoted as $h_{\text {stress }}\left(\vec{\chi}^{\prime \prime} \Rightarrow \vec{\chi}^{\prime}, t^{\prime}\right)$. The response of the stress-transfer pathway to any input of $i_{\text {stress }}\left(\vec{\chi}^{\prime \prime}, t^{\prime}\right)$ is the convolution of the stress with the impulse response and that becomes the intensity profile of the photon source as

$$
\begin{aligned}
q\left(\vec{\chi}^{\prime}, t^{\prime}\right) & =i_{\text {stress }}\left(\vec{\chi}^{\prime \prime}, t^{\prime}\right) \circledast h_{\text {stress }}\left(\vec{\chi}^{\prime \prime} \Rightarrow \vec{\chi}^{\prime}, t^{\prime}\right) \\
& =\int_{-\infty}^{\infty} i_{\text {stress }}\left(\vec{\chi}^{\prime \prime}, \beta\right) \cdot h_{\text {stress }}\left[\vec{\chi}^{\prime \prime} \Rightarrow \vec{\chi}^{\prime},\left(t^{\prime}-\beta\right)\right] d \beta .
\end{aligned}
$$

The photo-genesis in response to a bolus input represented by Eq. (3) is thus

$q_{\text {bolus }}\left(\vec{\chi}^{\prime}, t^{\prime}\right)=i_{\text {bolus }}\left(\vec{\chi}^{\prime \prime}, t^{\prime}\right) \circledast h_{\text {stress }}\left(\vec{\chi}^{\prime \prime} \Rightarrow \vec{\chi}^{\prime}, t^{\prime}\right)$.

The photo-genesis in response to a repetitive bolus input represented by Eq. (4) is then

$q_{\text {bolus }}^{\text {rep }}\left(\vec{\chi}^{\prime \prime}, t^{\prime}\right)=\sum_{n=0}^{N} q_{\text {bolus }}\left[\vec{\chi}^{\prime \prime},\left(t^{\prime}-n T\right)\right]$.

The photo-genesis in response to a step input represented by Eq. (5) is the following

$q_{\text {step }}\left(\vec{\chi}^{\prime}, t^{\prime}\right)=i_{\text {step }}\left(\vec{\chi}^{\prime \prime}, t^{\prime}\right) \circledast h_{\text {stress }}\left(\vec{\chi}^{\prime \prime} \Rightarrow \vec{\chi}^{\prime}, t^{\prime}\right)$.

The photo-genesis in response to a pulse input represented by Eq. (6) becomes

$q_{\text {pulse }}\left(\vec{\chi}^{\prime \prime}, t^{\prime}\right)=q_{\text {step }}\left(\vec{\chi}^{\prime \prime}, t^{\prime}\right)-q_{\text {step }}\left[\vec{\chi}^{\prime \prime},\left(t^{\prime}-\Delta T\right)\right]$.

The photo-genesis in response to a repetitive pulse input represented by Eq. (7) evolves as 


$$
\begin{aligned}
q_{\text {pulse }}^{\text {rep }}\left(\vec{\chi}^{\prime \prime}, t^{\prime}\right)= & \sum_{n=0}^{N} q_{\text {pulse }}\left[\vec{\chi}^{\prime \prime},\left(t^{\prime}-n T\right)\right] \\
& =\sum_{n=0}^{N}\left\{q_{\text {step }}\left[\vec{\chi}^{\prime \prime},\left(t^{\prime}-n T\right)\right]\right. \\
& \left.-q_{\text {step }}\left[\vec{\chi}^{\prime \prime},\left(t^{\prime}-\Delta T-n T\right)\right]\right\} .
\end{aligned}
$$

It is clear from Eqs. (9)-(13) that the photo-genic response to a complex stress may be adequately described by the linear combination of the responses to bolus and step stresses, which are practically applicable. Therefore, the following section restricts the model-analysis to the responses of stress-transfer pathways to a bolus stress of Eq. (3) and a step stress of Eq. (5).

\section{System models of the stress-transfer pathway}

The following analyses of photo-genetic kinetics are performed on stress-transfer pathways that are hypothesized to reveal the responses as illustrated in Fig. 3. $(A)$ refers to the first-order low-pass response that is considered as the simplest pathway to link the external stress and photogenesis exceeding that for the baseline spontaneous UPE. A stress-transfer pathway can also consist of two or more in-parallel pathways with each presenting a first-order low-pass response. (B) specifies a stress-transfer pathway that has a slowly varying kinetic rate governing the firstorder low-pass response. (C) represents a stress-transfer

$q_{\text {bolus }}^{1}\left(\vec{\chi}^{\prime}, t^{\prime}\right)=\left[A_{\text {stress }} \cdot \delta\left(\vec{\chi}-\vec{\chi}^{\prime \prime}\right) \cdot K_{01}\left(\vec{\chi}^{\prime \prime} \Rightarrow \vec{\chi}^{\prime}\right) \cdot K_{1 \mho}\left(\vec{\chi}^{\prime}\right)\right] \cdot e^{-K_{1 \mho}\left(\vec{\chi}^{\prime}\right) \cdot t^{\prime}} \cdot u\left(t^{\prime}\right)$.

And the response of this pathway to a step stress of magnitude $A_{\text {stress }}$ represented by Eq. (5) is

$q_{\text {step }}^{1}\left(\vec{\chi}^{\prime}, t^{\prime}\right)=\left[A_{\text {stress }} \cdot \delta\left(\vec{\chi}-\vec{\chi}^{\prime \prime}\right) \cdot K_{01}\left(\vec{\chi}^{\prime \prime} \Rightarrow \vec{\chi}^{\prime}\right)\right] \cdot\left[1-e^{-K_{1 \mho}\left(\vec{\chi}^{\prime}\right) \cdot t^{\prime}}\right] u\left(t^{\prime}\right)$. process that involves a single second-order low-pass pathway. (D) marks a stress-transfer process involving a single second-order band-pass pathway. The system structure of each pathway is detailed in the respective section.

\subsection{Stress transfer involving a single first-order low-pass pathway}

Compartmental analyses of the kinetics of various body functions have indicated low-pass pattern to be common for physiological or metabolic responses to external perturbations [40]. A first-order low-pass response is thus suitable to be considered as the simplest pathway linking the external stress and induced photo-genesis, as shown in Fig. 3a, which is the block-diagram equivalence of the simple stress-pass configuration speculated in Fig. 2. If denoting the respective Laplace transform (LT) of $q_{\text {phot }}\left(\vec{\chi}^{\prime}, t^{\prime}\right)$ and $i_{\text {stress }}\left(\vec{\chi}^{\prime \prime}, t^{\prime}\right)$ of Fig. 2 as $Q_{\text {phot }}\left(\vec{\chi}^{\prime}, s\right)$ and $l_{\text {stress }}\left(\vec{\chi}^{\prime \prime}, s\right)$, the transfer structure illustrated in $(A)$ results in the following transfer function

$\mathcal{H}_{\text {stress }}\left(\vec{\chi}^{\prime \prime} \Rightarrow \vec{\chi}^{\prime}, s\right)=\frac{Q_{\text {phot }}\left(\vec{\chi}^{\prime}, s\right)}{I_{\text {stress }}\left(\vec{\chi}^{\prime \prime}, s\right)}=K_{01}\left(\vec{\chi}^{\prime \prime} \Rightarrow \vec{\chi}^{\prime}\right) \cdot \frac{K_{1 \mho}\left(\vec{\chi}^{\prime}\right)}{s+K_{1 \mho}\left(\vec{\chi}^{\prime}\right)}$

which is a low-pass filter with a pass-band gain of $K_{01}\left(\vec{\chi}^{\prime \prime} \Rightarrow \vec{\chi}^{\prime}\right)$ and a cutoff frequency at $K_{1 \mho}\left(\vec{\chi}^{\prime}\right)$. The corresponding impulse response is:

$h_{\text {stress }}\left(\vec{\chi}^{\prime \prime} \Rightarrow \vec{\chi}^{\prime}, t^{\prime}\right)=K_{01}\left(\vec{\chi}^{\prime \prime} \Rightarrow \vec{\chi}^{\prime}\right) \cdot K_{1 \mho}\left(\vec{\chi}^{\prime}\right) \cdot e^{-K_{1 \mho}\left(\vec{\chi}^{\prime}\right) \cdot t^{\prime}} \cdot u\left(t^{\prime}\right)$.

The response of this pathway to a bolus stress of magnitude $A_{\text {stress }}$ represented by Eq. (3) is

\subsection{Stress transfer involving two or more in-parallel first-order low-pass pathways}

Induced PE in response to photic stress often decays hyperbolically. The hyperbolic pattern from mitochondria respiratory complex I in response to photic-stimulation is however shown to be fitted by 4 or 7 exponential functions [11]. A hyperbolic function that decays faster than an exponential function can be readily decomposed by using the basis of exponential functions. A system composed 
(A)

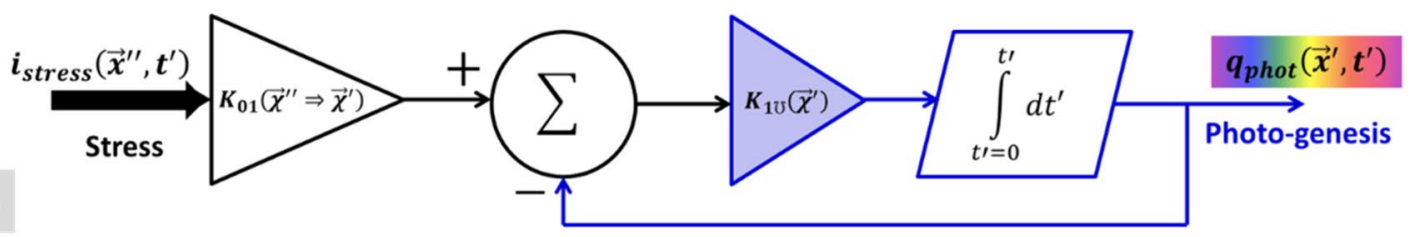

(B)

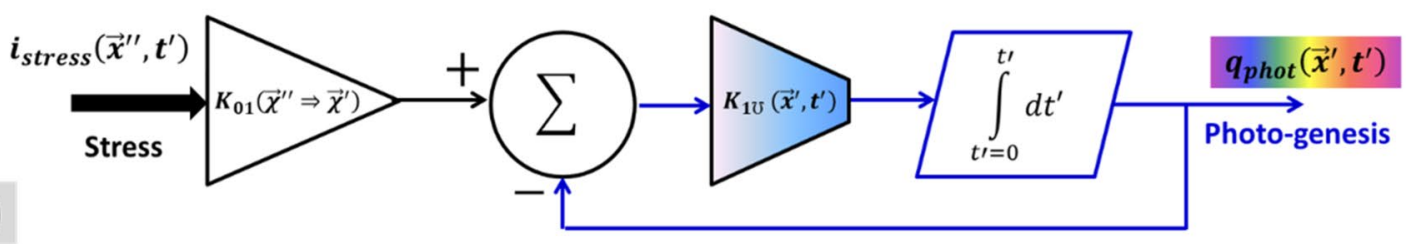

(C)
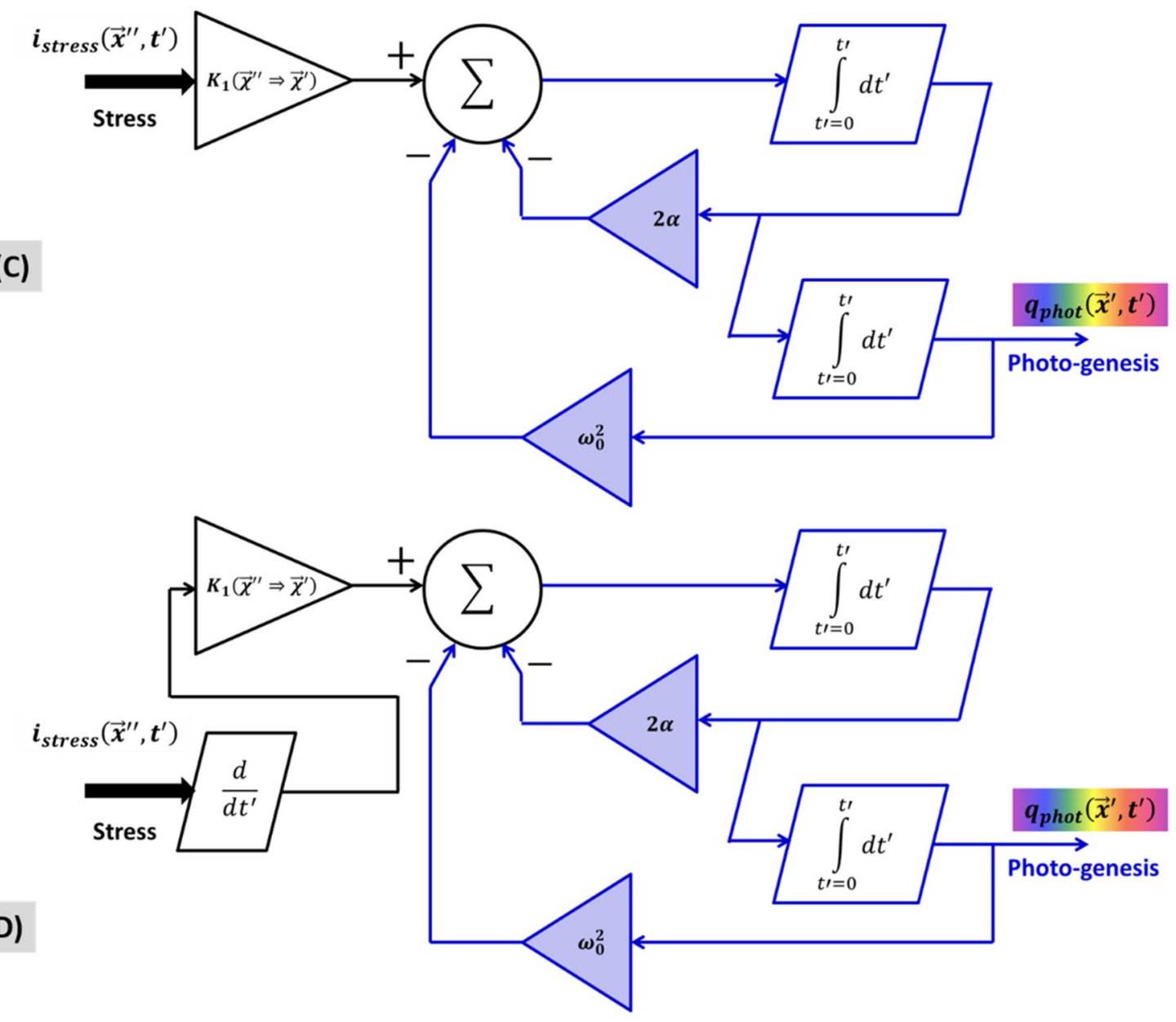

Fig. 3 Stress-transfer pathways that are modeled for the responses. a Stress transfer involving a first-order low-pass pathway having a fixed kinetic rate. b Stress transfer involving a first-order low-pass pathway having a slowly varying kinetic rate. c Stress transfer

involving a second-order low-pass pathway having fixed kinetic or feedback rates. d Stress transfer involving a second-order bandpass pathway having fixed kinetic or feedback rates

of multiple in-parallel first-order low-pass pathways can thus manifest responses that are superficially hyperbolic.

A stress-transfer pathway consisting of two or more inparallel pathways, each presenting a first-order low-pass response as is illustrated in (A) has a transfer function of: 
$\mathcal{H}_{\text {stress }}\left(\vec{\chi}^{\prime \prime} \Rightarrow \vec{\chi}^{\prime}, s\right)=\frac{Q_{\text {phot }}\left(\vec{\chi}^{\prime}, s\right)}{l_{\text {stress }}\left(\vec{\chi}^{\prime \prime}, s\right)}=\sum_{m=1}^{M} K_{0 m}\left(\vec{\chi}^{\prime \prime} \Rightarrow \vec{\chi}^{\prime}\right) \cdot \frac{K_{m \mho}\left(\vec{\chi}^{\prime}\right)}{s+K_{m \mho}\left(\vec{\chi}^{\prime}\right)}, \quad(M \geq 2)$

that corresponds to an impulse response of:

$h_{\text {stress }}\left(\vec{\chi}^{\prime \prime} \Rightarrow \vec{\chi}^{\prime}, t^{\prime}\right)=\sum_{m=1}^{M}\left[K_{0 m}\left(\vec{\chi}^{\prime \prime} \Rightarrow \vec{\chi}^{\prime}\right) \cdot K_{m \mho}\left(\vec{\chi}^{\prime}\right) \cdot e^{-K_{m \mho}\left(\vec{\chi}^{\prime}\right) \cdot t^{\prime}}\right] \cdot u\left(t^{\prime}\right)$.

The response of this pathway to a bolus stress of mag-

nitude $A_{\text {stress }}$ represented by Eq. (3) is

$q_{\text {bolus }}^{M}\left(\vec{\chi}^{\prime}, t^{\prime}\right)=A_{\text {stress }} \cdot \delta\left(\vec{\chi}-\vec{\chi}^{\prime \prime}\right) \cdot \sum_{m=1}^{M}\left[K_{0 m}\left(\vec{\chi}^{\prime \prime} \Rightarrow \vec{\chi}^{\prime}\right) \cdot K_{m \mho}\left(\vec{\chi}^{\prime}\right) \cdot e^{-K_{m \mho}\left(\vec{\chi}^{\prime}\right) \cdot t^{\prime}}\right] \cdot u\left(t^{\prime}\right)$.

The response of this pathway to a step stress of magni-

tude $A_{\text {stress }}$ represented by Eq. (5) is

$q_{\text {step }}^{M}\left(\vec{\chi}^{\prime}, t^{\prime}\right)=A_{\text {stress }} \cdot \delta\left(\vec{\chi}-\vec{\chi}^{\prime \prime}\right) \cdot \sum_{m=1}^{M}\left\{K_{0 m}\left(\vec{\chi}^{\prime \prime} \Rightarrow \vec{\chi}^{\prime}\right) \cdot\left[1-e^{-K_{m \mho}\left(\vec{\chi}^{\prime}\right) \cdot t^{\prime}}\right]\right\} u\left(t^{\prime}\right)$.

\subsection{Stress transfer involving single first-order low-pass pathway with slowly varying kinetic rate}

A first-order system with a time-varying kinetic rate has been shown applicable to a number of biological rate changes including fluorescence decay lasting less than 35 ns [41]. Similar kinetics governed by slowly time-varying rates has been found for delayed fluorescence resulting from triplet-triplet annihilation in polyphenyl quinoxalines in frozen solutions or films, and phosphorescence intensity decay of strontium sulfide with the delay recorded over 2000s [42].
The varying kinetic rate fitting the biological responses well has been associated with a lifetime distribution that follows a gamma function. It has been suggested that this type of slowly varying kinetics within a first-order response may be applicable to a great variety of other biological systems, including induced PE excited by a stress [42]. A stress-transfer pathway that has a slowly varying kinetic rate governing the first-order low-pass response is shown in Fig. 3b. The system represented by $(B)$ differs from the one by $(A)$ in only one aspect, the transition between the summer and the integrator is controlled at a slowly varying kinetic rate in lieu of a constant kinetic rate. The equivalent impulse response of this system can be approximated as

$h_{\text {stress }}\left(\vec{\chi}^{\prime \prime} \Rightarrow \vec{\chi}^{\prime}, t^{\prime}\right)=K_{01}\left(\vec{\chi}^{\prime \prime} \Rightarrow \vec{\chi}^{\prime}\right) \cdot K_{1 \mho}\left(\vec{\chi}^{\prime}, t^{\prime}\right) \cdot e^{-K_{1 \mho}\left(\vec{\chi}^{\prime}, t^{\prime}\right) \cdot t^{\prime}} \cdot u\left(t^{\prime}\right)$.

The response of this pathway to a bolus stress of magnitude $A_{\text {stress }}$ represented by Eq. (3) is

$q_{\text {bolus }}^{\tau\left(t^{\prime}\right)}\left(\vec{\chi}^{\prime}, t^{\prime}\right)=\left[A_{\text {stress }} \cdot \delta\left(\vec{\chi}-\vec{\chi}^{\prime \prime}\right) \cdot K_{01}\left(\vec{\chi}^{\prime \prime} \Rightarrow \vec{\chi}^{\prime}\right) \cdot K_{1 \mho}\left(\vec{\chi}^{\prime}, t^{\prime}\right)\right] \cdot e^{-K_{1 \mho}\left(\vec{\chi}^{\prime}, t^{\prime}\right) \cdot t^{\prime}} \cdot u\left(t^{\prime}\right)$.

The response of this pathway to a step stress of magnitude $A_{\text {stress }}$ of Eq. (5) can be found by convolving 
$h_{\text {stress }}\left(\vec{\chi}^{\prime \prime} \Rightarrow \vec{\chi}^{\prime}, t^{\prime}\right)$ of Eq. (19) with $i_{\text {step }}\left(\vec{\chi}^{\prime \prime}, t^{\prime}\right)$ of Eq. (5)

and that leads to

$q_{\text {step }}^{\tau\left(t^{\prime}\right)}\left(\vec{\chi}^{\prime}, t^{\prime}\right)=\left[A_{\text {stress }} \cdot \delta\left(\vec{\chi}-\vec{\chi}^{\prime \prime}\right) \cdot K_{01}\left(\vec{\chi}^{\prime \prime} \Rightarrow \vec{\chi}^{\prime}\right)\right] \cdot\left[1-e^{-K_{1 v}} \overline{\left(\vec{x}^{\prime}, t^{\prime}\right) \cdot t^{\prime}}\right] u\left(t^{\prime}\right)$.

\subsection{Stress transfer involving a second-order low-pass pathway}

A stress-transfer process that involves a single secondorder low-pass pathway is illustrated in Fig. 3c. The system contains two kinetic rates in terms of how the negative feedback is taken from, respectively, the output and the first-order derivative of the output. The amount of the negative feedback that is taken from the first-order derivative of the output is denoted as $2 \alpha$, whereas the amount of the negative feedback that is taken directly from the output is denoted as $\omega_{0}^{2}$. The system responds in four ways to an input, depending upon the relative scale between $\alpha$ and $\omega_{0}$.

\subsubsection{The response corresponding to ( $\alpha=0)$ that is referred to as the un-damped case}

The transfer function is

$\mathcal{H}_{\text {stress }}\left(\vec{\chi}^{\prime \prime} \Rightarrow \vec{\chi}^{\prime}, s\right)=K_{1}\left(\vec{\chi}^{\prime \prime} \Rightarrow \vec{\chi}^{\prime}\right) \cdot \frac{1}{s^{2}+\omega_{0}^{2}}$

that corresponds to an impulse response of

$h_{\text {stress }}\left(\vec{\chi}^{\prime \prime} \Rightarrow \vec{\chi}^{\prime}, t^{\prime}\right)=K_{01}\left(\vec{\chi}^{\prime \prime} \Rightarrow \vec{\chi}^{\prime}\right) \cdot \frac{1}{\omega_{0}} \cdot \sin \left(\omega_{0} t^{\prime}\right) \cdot u\left(t^{\prime}\right)$.

$q_{\text {bolus }}^{\alpha<\omega_{0}}\left(\vec{\chi}^{\prime \prime} \Rightarrow \vec{\chi}^{\prime}, t^{\prime}\right)=A_{\text {stress }} \cdot K_{01}\left(\vec{\chi}^{\prime \prime} \Rightarrow \vec{\chi}^{\prime}\right) \cdot \frac{1}{\omega_{d}} \cdot e^{-\alpha t^{\prime}} \cdot \sin \left(\omega_{d} t^{\prime}\right) \cdot u\left(t^{\prime}\right)$.
The response of this pathway to a bolus stress of magnitude $A_{\text {stress }}$ represented by Eq. (3) is

$q_{\text {bolus }}^{\alpha=0}\left(\vec{\chi}^{\prime \prime} \Rightarrow \vec{\chi}^{\prime}, t^{\prime}\right)=A_{\text {stress }} \cdot K_{01}\left(\vec{\chi}^{\prime \prime} \Rightarrow \vec{\chi}^{\prime}\right) \cdot \frac{1}{\omega_{0}} \cdot \sin \left(\omega_{0} t^{\prime}\right) \cdot u\left(t^{\prime}\right)$.

The response of this pathway to a step stress of magnitude $A_{\text {stress }}$ represented by Eq. (5) is

$q_{\text {step }}^{\alpha=0}\left(\vec{\chi}^{\prime \prime} \Rightarrow \vec{\chi}^{\prime}, t^{\prime}\right)=A_{\text {stress }} \cdot K_{01}\left(\vec{\chi}^{\prime \prime} \Rightarrow \vec{\chi}^{\prime}\right) \cdot \frac{1}{\omega_{0}^{2}} \cdot\left[1-\cos \left(\omega_{0} t^{\prime}\right)\right] \cdot u\left(t^{\prime}\right)$.

\subsubsection{The response corresponding to $\left(0<\alpha<\omega_{0}\right)$ that is referred to as the under-damped case}

The transfer function is

$\mathcal{H}_{\text {stress }}\left(\vec{\chi}^{\prime \prime} \Rightarrow \vec{\chi}^{\prime}, s\right)=K_{01}\left(\vec{\chi}^{\prime \prime} \Rightarrow \vec{\chi}^{\prime}\right) \cdot \frac{1}{\left(s+\alpha-j \omega_{d}\right)\left(s+\alpha+j \omega_{d}\right)}$.

The following notation is useful:

$\omega_{d}=\sqrt{\left|\alpha^{2}-\omega_{0}^{2}\right|}$.

Equation (29) corresponds to an impulse response of

$h_{\text {stress }}\left(\vec{\chi}^{\prime \prime} \Rightarrow \vec{\chi}^{\prime}, t^{\prime}\right)=K_{01}\left(\vec{\chi}^{\prime \prime} \Rightarrow \vec{\chi}^{\prime}\right) \cdot \frac{1}{\omega_{d}} \cdot e^{-\alpha t^{\prime}} \cdot \sin \left(\omega_{d} t^{\prime}\right) \cdot u\left(t^{\prime}\right)$.

The response of this pathway to a bolus stress of magnitude $A_{\text {stress }}$ represented by Eq. (3) is 
The response of this pathway to a step stress of magnitude $A_{\text {stress }}$ represented by Eq. (5) is

$$
\begin{gathered}
q_{\text {step }}^{\alpha<\omega_{0}}\left(\vec{\chi}^{\prime \prime} \Rightarrow \vec{\chi}^{\prime}, t^{\prime}\right)=A_{\text {stress }} \cdot K_{01}\left(\vec{\chi}^{\prime \prime} \Rightarrow \vec{\chi}^{\prime}\right) \\
\cdot \frac{1}{\omega_{d}^{2}\left[1+\left(\frac{\alpha}{\omega_{d}}\right)^{2}\right]}\left\{1-\sqrt{1+\left(\frac{\alpha}{\omega_{d}}\right)^{2}} \cdot e^{-\alpha t^{\prime}}\right. \\
\left.\cdot \cos \left(\omega_{d} t^{\prime}-\tan ^{-1}\left(\frac{\alpha}{\omega_{d}}\right)\right)\right\} \cdot u\left(t^{\prime}\right) .
\end{gathered}
$$

\subsubsection{The response corresponding to $\left(\alpha=\omega_{0}\right)$ that is referred to as the critically damped case}

The transfer function is

$$
\mathcal{H}_{\text {stress }}\left(\vec{\chi}^{\prime \prime} \Rightarrow \vec{\chi}^{\prime}, s\right)=K_{01}\left(\vec{\chi}^{\prime \prime} \Rightarrow \vec{\chi}^{\prime}\right) \cdot \frac{1}{(s+\alpha)^{2}}
$$

$h_{\text {stress }}\left(\vec{\chi}^{\prime \prime} \Rightarrow \vec{\chi}^{\prime}, t^{\prime}\right)=K_{01}\left(\vec{\chi}^{\prime \prime} \Rightarrow \vec{\chi}^{\prime}\right) \frac{1}{2 \omega_{d}} \cdot e^{-\left(\alpha-\omega_{d}\right) t^{\prime}} \cdot\left[1-e^{-2 \omega_{d} t^{\prime}}\right] \cdot u\left(t^{\prime}\right)$.

The response of this pathway to a bolus stress of magnitude $A_{\text {stress }}$ represented by Eq. (3) is

$q_{\text {bolus }}^{\alpha>\omega_{0}}\left(\vec{\chi}^{\prime \prime} \Rightarrow \vec{\chi}^{\prime}, t^{\prime}\right)=A_{\text {stress }} \cdot K_{01}\left(\vec{\chi}^{\prime \prime} \Rightarrow \vec{\chi}^{\prime}\right) \cdot \frac{1}{2 \omega_{d}} \cdot e^{-\left(\alpha-\omega_{d}\right) t^{\prime}} \cdot\left[1-e^{-2 \omega_{d} t^{\prime}}\right] \cdot u\left(t^{\prime}\right)$.

The response of this pathway to a step stress of magnitude $A_{\text {stress }}$ represented by Eq. (5) is

$q_{\text {step }}^{\alpha=\omega_{0}}\left(\vec{\chi}^{\prime \prime} \Rightarrow \vec{\chi}^{\prime}, t^{\prime}\right)=A_{\text {stress }} \cdot K_{01}\left(\vec{\chi}^{\prime \prime} \Rightarrow \vec{\chi}^{\prime}\right) \cdot \frac{1}{\alpha^{2}\left[1-\left(\frac{\omega_{d}}{\alpha}\right)^{2}\right]} \cdot\left\{1-\frac{1}{2 \omega_{d}}\left[\left(\alpha+\omega_{d}\right) e^{-\left(\alpha-\omega_{d}\right) t^{\prime}}-\left(\alpha-\omega_{d}\right) e^{-\left(\alpha+\omega_{d}\right) t^{\prime}}\right]\right\} \cdot u\left(t^{\prime}\right)$. 


\subsection{Stress transfer involving a second-order band-pass pathway}

A stress-transfer process involving a single second-order band-pass pathway is shown in Fig. 3d. The system of (D) assimilates the one of (C) in terms of the two kinetic rates determining the negative feedback taken, respectively, from the output and the first-order derivative of the output. The system of (D) differs from (C) in that the firstorder derivative of the input, not the input itself, is passed through the system. The system responds to an input in four ways, depending upon the relative scale between $\alpha$ and $\omega_{0}$, by referring to Eq. (29) for $\omega_{d}$.

\subsubsection{The response corresponding to $(\alpha=0)$ that is referred to as the un-damped case}

The transfer function of the pathway is

$\mathcal{H}_{\text {stress }}\left(\vec{\chi}^{\prime \prime} \Rightarrow \vec{\chi}^{\prime}, s\right)=K_{01}\left(\vec{\chi}^{\prime \prime} \Rightarrow \vec{\chi}^{\prime}\right) \cdot \frac{s}{s^{2}+\omega_{0}^{2}}$.

The corresponding impulse response is

$h_{\text {stress }}\left(\vec{\chi}^{\prime \prime} \Rightarrow \vec{\chi}^{\prime}, t^{\prime}\right)=A_{\text {stress }} \cdot K_{01}\left(\vec{\chi}^{\prime \prime} \Rightarrow \vec{\chi}^{\prime}\right) \cdot \cos \left(\omega_{0} t^{\prime}\right) \cdot u\left(t^{\prime}\right)$.
The response of this pathway to a bolus stress of magnitude $A_{\text {stress }}$ represented by Eq. (3) is

$q_{\text {step }}^{\alpha=0}\left(\vec{\chi}^{\prime \prime} \Rightarrow \vec{\chi}^{\prime}, t^{\prime}\right)=A_{\text {stress }} \cdot K_{01}\left(\vec{\chi}^{\prime \prime} \Rightarrow \vec{\chi}^{\prime}\right) \cdot \cos \left(\omega_{0} t^{\prime}\right) \cdot u\left(t^{\prime}\right)$.

The response of this pathway to a step stress of magnitude $A_{\text {stress }}$ represented by Eq. (5) is

$q_{\text {step }}^{\alpha=0}\left(\vec{\chi}^{\prime \prime} \Rightarrow \vec{\chi}^{\prime}, t^{\prime}\right)=A_{\text {stress }} \cdot K_{01}\left(\vec{\chi}^{\prime \prime} \Rightarrow \vec{\chi}^{\prime}\right) \cdot \frac{1}{\omega_{0}} \cdot \sin \left(\omega_{0} t^{\prime}\right) \cdot u\left(t^{\prime}\right)$.

\subsubsection{The response corresponding to $\left(0<\alpha<\omega_{0}\right)$ that is referred to as the under-damped case}

The transfer function of the pathway is

$$
\mathcal{H}_{\text {stress }}\left(\vec{\chi}^{\prime \prime} \Rightarrow \vec{\chi}^{\prime}, s\right)=K_{01}\left(\vec{\chi}^{\prime \prime} \Rightarrow \vec{\chi}^{\prime}\right) \cdot \frac{s}{\left(s+\alpha-j \omega_{d}\right)\left(s+\alpha+j \omega_{d}\right)} \text {. }
$$

The corresponding impulse response is

$$
\begin{aligned}
& h_{\text {stress }}\left(\vec{\chi}^{\prime \prime} \Rightarrow \vec{\chi}^{\prime}, t^{\prime}\right)=K_{01}\left(\vec{\chi}^{\prime \prime} \Rightarrow \vec{\chi}^{\prime}\right) \\
& \cdot \sqrt{1+\left(\frac{\alpha}{\omega_{d}}\right)^{2}} \cdot e^{-\alpha t^{\prime}} \cdot \cos \left[\omega_{d} t^{\prime}-\tan ^{-1}\left(\frac{\alpha}{\omega_{d}}\right)\right] \cdot u\left(t^{\prime}\right) .
\end{aligned}
$$

The response of this pathway to a bolus stress of magnitude $A_{\text {stress }}$ represented by Eq. (3) is

$q_{\text {bolus }}^{\alpha<\omega_{0}}\left(\vec{\chi}^{\prime \prime} \Rightarrow \vec{\chi}^{\prime}, t^{\prime}\right)=A_{\text {stress }} \cdot K_{01}\left(\vec{\chi}^{\prime \prime} \Rightarrow \vec{\chi}^{\prime}\right) \cdot \sqrt{1+\left(\frac{\alpha}{\omega_{d}}\right)^{2}} \cdot e^{-\alpha t^{\prime}} \cdot \cos \left[\omega_{d} t^{\prime}-\tan ^{-1}\left(\frac{\alpha}{\omega_{d}}\right)\right] \cdot u\left(t^{\prime}\right)$.

The response of this pathway to a step stress of magnitude $A_{\text {stress }}$ represented by Eq. (5) is

$q_{\text {step }}^{\alpha<\omega_{0}}\left(\vec{\chi}^{\prime \prime} \Rightarrow \vec{\chi}^{\prime}, t^{\prime}\right)=A_{\text {stress }} \cdot K_{01}\left(\vec{\chi}^{\prime \prime} \Rightarrow \vec{\chi}^{\prime}\right) \cdot \frac{1}{\omega_{d}} \cdot e^{-\alpha t^{\prime}} \cdot \sin \left(\omega_{d} t^{\prime}\right) \cdot u\left(t^{\prime}\right)$. 

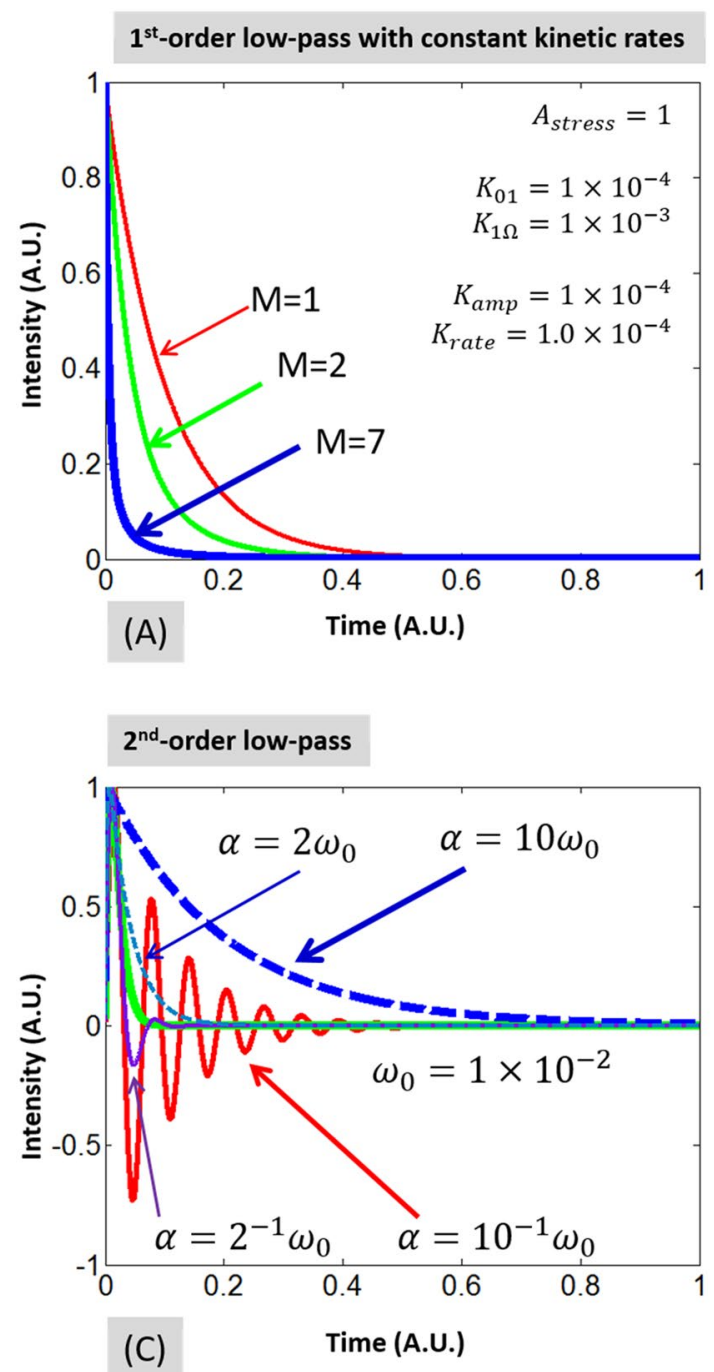

Fig. 4 The responses of the hypothesized stress-transfer pathways to a bolus input. a The responses characterized by single or multiple in-parallel first-order low-pass systems with constant kinetic rates. $\mathbf{b}$ The responses characterized by single first-order low-pass system with slowly varying kinetic rate. $\mathbf{c}$ The responses character-

\subsubsection{The response corresponding to $\left(\alpha=\omega_{0}\right)$ that is referred to as the critically damped case}

The transfer function of the pathway is

$$
\mathcal{H}_{\text {stress }}\left(\vec{\chi}^{\prime \prime} \Rightarrow \vec{\chi}^{\prime}, s\right)=K_{01}\left(\vec{\chi}^{\prime \prime} \Rightarrow \vec{\chi}^{\prime}\right) \cdot \frac{s}{(s+\alpha)^{2}} \text {. }
$$

The corresponding impulse response is

$$
h_{\text {stress }}\left(\vec{\chi}^{\prime \prime} \Rightarrow \vec{\chi}^{\prime}, t^{\prime}\right)=K_{01}\left(\vec{\chi}^{\prime \prime} \Rightarrow \vec{\chi}^{\prime}\right) \cdot e^{-\alpha t^{\prime}} \cdot\left(1-\alpha t^{\prime}\right) \cdot u\left(t^{\prime}\right) \text {. }
$$

The response of this pathway to a bolus stress of magnitude $A_{\text {stress }}$ represented by Eq. (3) is
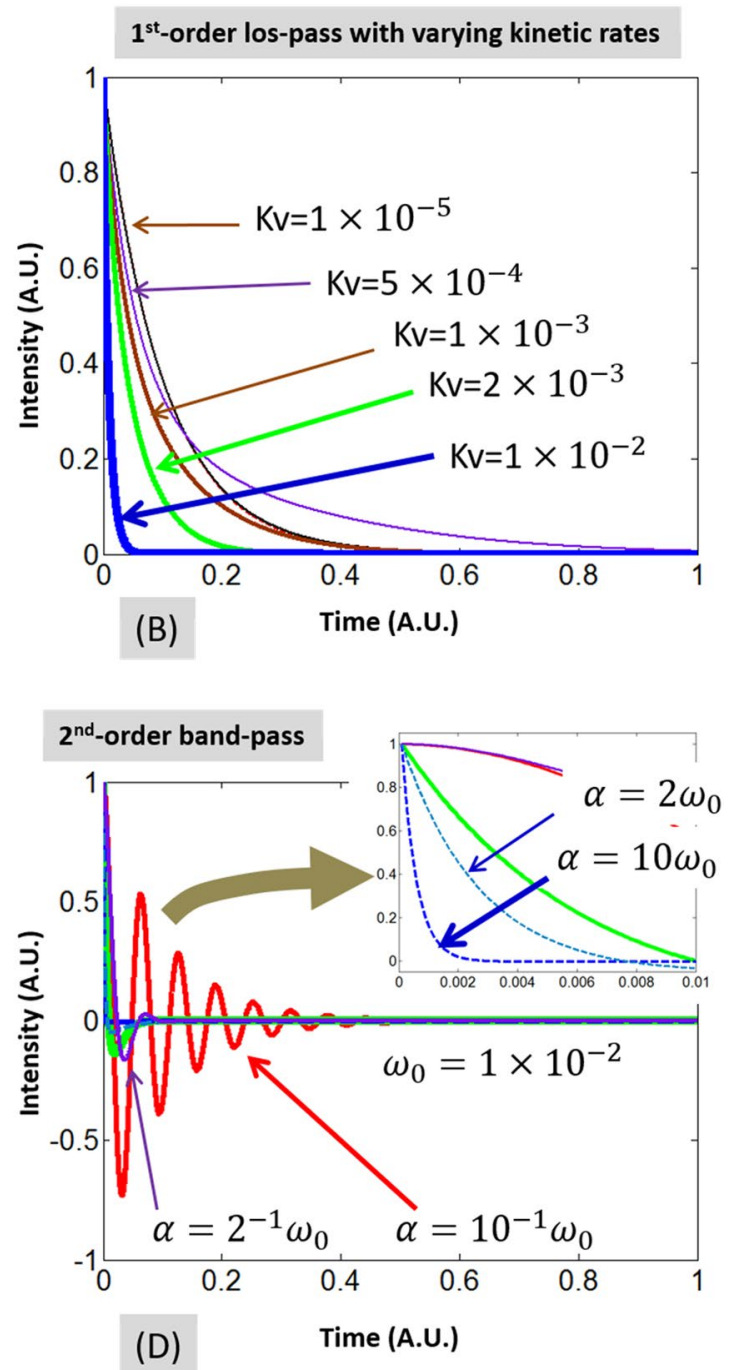

ized by a second-order low-pass system with fixed kinetic rates but different nonzero damping factors. $\mathbf{d}$ The responses characterized by a second-order band-pass system with fixed kinetic rates but different nonzero damping factors

$q_{\text {bolus }}^{\alpha=\omega_{0}}\left(\vec{\chi}^{\prime \prime} \Rightarrow \vec{\chi}^{\prime}, t^{\prime}\right)=A_{\text {stress }} \cdot K_{01}\left(\vec{\chi}^{\prime \prime} \Rightarrow \vec{\chi}^{\prime}\right) \cdot e^{-\alpha t^{\prime}} \cdot\left(1-\alpha t^{\prime}\right) \cdot u\left(t^{\prime}\right)$.

The response of this pathway to a step stress of magnitude $A_{\text {stress }}$ represented by Eq. (5) is

$q_{\text {step }}^{\alpha=\omega_{0}}\left(\vec{\chi}^{\prime \prime} \Rightarrow \vec{\chi}^{\prime}, t^{\prime}\right)=A_{\text {stress }} \cdot K_{01}\left(\vec{\chi}^{\prime \prime} \Rightarrow \vec{\chi}^{\prime}\right) \cdot t^{\prime} \cdot e^{-\alpha t^{\prime}} \cdot u\left(t^{\prime}\right)$.

\subsubsection{The response corresponding to $\left(\alpha>\omega_{0}\right)$ that is referred to as the over-damped case}

The transfer function of the pathway is 
$\mathcal{H}_{\text {stress }}\left(\vec{\chi}^{\prime \prime} \Rightarrow \vec{\chi}^{\prime}, s\right)=K_{01}\left(\vec{\chi}^{\prime \prime} \Rightarrow \vec{\chi}^{\prime}\right) \cdot \frac{s}{\left(s+\alpha-\omega_{d}\right)\left(s+\alpha+\omega_{d}\right)}$.

The corresponding impulse response is

$h_{\text {stress }}\left(\vec{\chi}^{\prime \prime} \Rightarrow \vec{\chi}^{\prime}, t^{\prime}\right)=K_{01}\left(\vec{\chi}^{\prime \prime} \Rightarrow \vec{\chi}^{\prime}\right) \cdot \frac{1}{2 \omega_{d}} \cdot\left[\left(\alpha+\omega_{d}\right) e^{-\left(\alpha+\omega_{d}\right) t^{\prime}}-\left(\alpha-\omega_{d}\right) e^{-\left(\alpha-\omega_{d}\right) t^{\prime}}\right] \cdot u\left(t^{\prime}\right)$.

The response of this pathway to a bolus stress of mag-

nitude $A_{\text {stress }}$ represented by Eq. (3) is

$q_{\text {bolus }}^{\alpha>\omega_{0}}\left(\vec{\chi}^{\prime \prime} \Rightarrow \vec{\chi}^{\prime}, t^{\prime}\right)=A_{\text {stress }} \cdot K_{01}\left(\vec{\chi}^{\prime \prime} \Rightarrow \vec{\chi}^{\prime}\right) \cdot \frac{1}{2 \omega_{d}} \cdot\left[\left(\alpha+\omega_{d}\right) e^{-\left(\alpha+\omega_{d}\right) t^{\prime}}-\left(\alpha-\omega_{d}\right) e^{-\left(\alpha-\omega_{d}\right) t^{\prime}}\right] \cdot u\left(t^{\prime}\right)$.
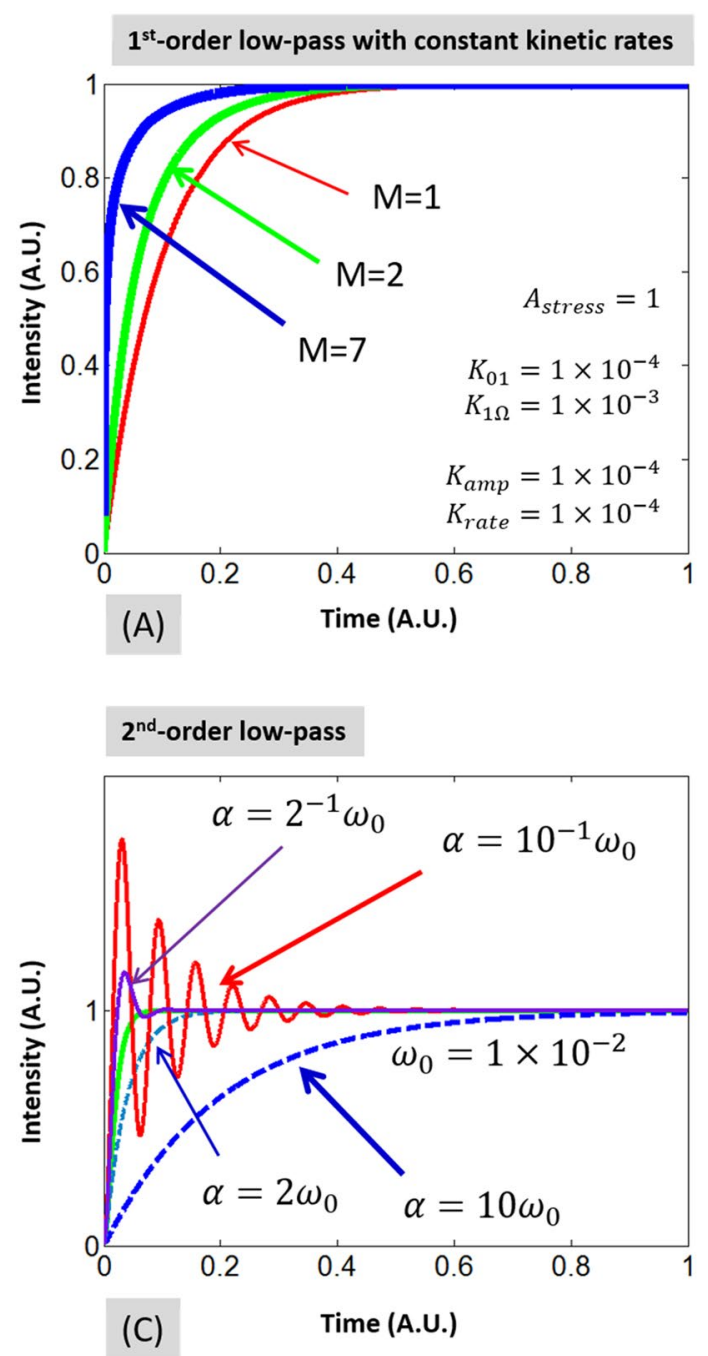

Fig. 5 The responses of the hypothesized stress-transfer pathways to a step input. a The responses characterized by single or multiple in-parallel first-order low-pass systems with constant kinetic rates. b The responses characterized by single first-order low-pass system with slowly varying kinetic rate. $\mathbf{c}$ The responses characterized by
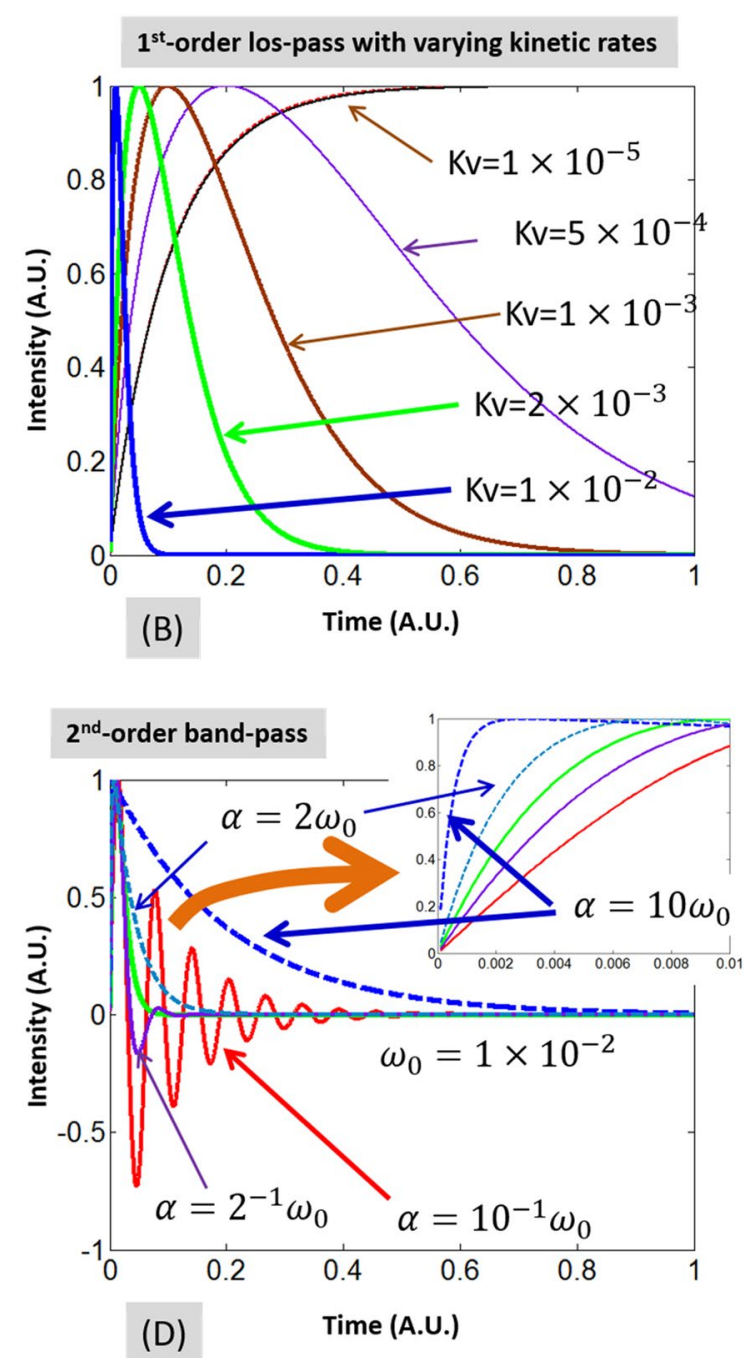

a second-order low-pass system with fixed kinetic rates but different nonzero damping factors. $\mathbf{d}$ The responses characterized by a second-order band-pass system with fixed kinetic rates but different nonzero damping factors 
The response of this pathway to a step stress of magnitude $A_{\text {stress }}$ represented by Eq. (5) is change over time, and that rate is always affected by the temporal resolution of the measurement and the total

$q_{\text {step }}^{\alpha>\omega_{0}}\left(\vec{\chi}^{\prime \prime} \Rightarrow \vec{\chi}^{\prime}, t^{\prime}\right)=A_{\text {stress }} \cdot K_{01}\left(\vec{\chi}^{\prime \prime} \Rightarrow \vec{\chi}^{\prime}\right) \cdot \frac{1}{2 \omega_{d}} \cdot e^{-\left(\alpha-\omega_{d}\right) t^{\prime}} \cdot\left[1-e^{-2 \omega_{d} t^{\prime}}\right] \cdot u\left(t^{\prime}\right)$.

\section{Numerical evaluations of the stress-transfer responses to bolus and step inputs}

This section numerically assesses the responses of the hypothesized stress-transfer pathways to a bolus input and a step input. The numerical assessment is performed for the following cases. Equations (16), (20), and (23) are implemented for assessing the responses of the stresstransfer processes hypothesized to consist of first-order low-pass pathways with fixed kinetic rates or a time-varying kinetic rate, to a bolus stress. Equation (20) is implemented for the system to contain two in-parallel first-order pathways and 7 in-parallel first-order pathways. Equations (27), (32), (36), and (40) are implemented for assessing the responses of the stress-transfer processes hypothesized to contain a single second-order low-pass pathway with fixed kinetic rates but differing in damping, to a bolus stress. Equations (44), (48), (52), and (56) are implemented for assessing the responses of the stress-transfer processes hypothesized to contain a single second-order band-pass pathway with fixed kinetic rates but differing in damping, to a bolus stress. Equations (17), (21), and (24) are implemented for assessing the responses of the stress-transfer process hypothesized to consist of first-order low-pass pathways with fixed kinetic rates or a time-varying kinetic rate, to a step stress. Equation (21) is implemented for the system to contain two in-parallel first-order pathways and 7 in-parallel first-order pathways. Equations (28), (33), (37), and (41) are implemented for assessing the responses of the stress-transfer processes hypothesized to contain a single second-order low-pass pathway with fixed kinetic rates but differing in the damping, to a step stress. Equations (45), (49), (53), and (57) are implemented to assess the responses of the stress-transfer processes hypothesized to contain a single second-order band-pass pathway with fixed kinetic rates but differing in damping, to a step stress.

The equations to be numericized are implemented according to the following principles: (1) The kinetics is evaluated over a timescale of [0 1] with a temporal resolution of $10^{-4}$. This resolution corresponds to measuring 10,000 times over the duration of the acquisition or covering a total of 4 orders of magnitude in time. This relative scaling is applicable to the decay kinetics of induced $P E$ because what is presented is the rate of the intensity duration of the data acquisition. (2) $K_{01}=1$ is assigned for all processes involving first-order low-pass pathways. (3) A total of 7 stages are configured for the stress-transfer processes hypothesized to contain more than two inparallel first-order low-pass pathways. This setting aligns with the case reporting that the kinetics of the induced $\mathrm{PE}$ in response to photo-illumination is found to be fitted with a total of 4 or 7 exponential functions [11]. (4) The kinetic rate of the single first-order low-pass system is set as $K_{1 \Omega}=10$, or 10 times in magnitude of the total duration of evaluation. This is equivalent to having the time constant of the single first-order low-pass system to be $10^{-1}$ of the total duration of evaluation. (5) For a process that is hypothesized to contain two or more first-order lowpass pathways with fixed kinetic rates, the kinetic rate of the last stage of the multiple-exponential system is set as $K_{M \Omega}=10$, i.e., 10 times in magnitude of the total duration of evaluation. The kinetic rates of the other pathways of $m \in[1, M-1]$ are defined as $K_{m \Omega}=K_{M \Omega} \cdot \exp (M-m)$. This exponential reduction of the kinetic rates of the pathways of 1 to $M$ is equivalent to having the time constants of the pathways of 1 to $M$ to increase at an exponential rate. (6) For a process hypothesized to contain a first-order low-pass pathway having a time-varying kinetic rate, the terminal kinetic rate of the system which is the kinetic rate at the end of the duration of evaluation is set as $K_{1 \Omega}($ end $)=10$, i.e., 10 times in magnitude of the total duration of evaluation. The kinetic rate that varies over time is defined as $K_{1 \Omega}(t)=K_{1 \Omega}($ end $) \cdot \exp \left[K_{v} \cdot t\right]$, where $K_{v}$ represents how fast the kinetic rate is changed. This makes the kinetic rate of the hypothesized pathway to decrease exponentially or equivalently the response of the pathway to slow down at an exponential rate. (7) For a process containing a single second-order pathway, the un-damped or zero-damping responses are not evaluated because it is simply the limiting case of under-damped response. The under-damped responses are evaluated at two exemplary cases of $\alpha=10^{-1} \omega_{0}$ and $\alpha=2^{-1} \omega_{0}$, respectively. The overdamped responses are evaluated also at two exemplary cases, corresponding to $\alpha=2 \omega_{0}$ and $\alpha=10 \omega_{0}$, respectively. The critically damped response is evaluated at a single frequency of $\omega_{0}=100$ which is also the frequency assigned to all cases. 


\subsection{The responses of the hypothesized stress-transfer pathways to a bolus input}

The responses of the hypothesized model pathways of stress transfer to a bolus input are displayed in Fig. 4. The (A) specifies the bolus responses of the stress-transfer pathways characterized by single or multiple in-parallel first-order low-pass systems having constant kinetic rates. The " $M$ " number indicates the number of first-order lowpass system hypothesized to be involved in the stresstransfer pathway. $M=1$ corresponds to a kinetic decay defined by a single exponential function. $M=2$ corresponds to a kinetic decay defined by a combination of two exponential functions. According to Eq. (20), the ratio of the initial intensities of these two exponential functions is identical to the ratio of the kinetic rates of the two exponential functions. $M=7$ corresponds to a kinetic decay defined by a combination of seven exponential functions. The initial intensities and the kinetic rates of these exponential functions decrease exponentially according to the aforementioned principles. As the number of exponential functions increases, the decay pattern deviates more and more from the single-exponential curve and resembles more of a hyperbolic curve. The initial decay becomes faster and the tail grounds at much slower rate. The transition between the faster initial decay and the slower later lag is more pronounced as the number of the first-order low-pass pathways increases.

The (B) is specific to the bolus responses of the stresstransfer pathways characterized by single first-order lowpass systems with a time-varying kinetic rate. The kinetic rate decreases exponentially according to the aforementioned principles. The decay pattern corresponding to $K_{v}=0.00001$ is indistinguishable from the pattern of single first-order low-pass response defined by a fixed kinetic rate that is identical to the initial value of the kinetic rate of this system with a time-varying kinetics. As the kinetic rate varies faster, the decay pattern deviates more and more from the single-exponential curve and resembles more of a hyperbolic one. The initial decay becomes faster and the grounding tail becomes longer. The transition between the fast initial decay and the slow later lag is more pronounced as the rate of change of the kinetic constant increases.

The $(C)$ is specific to the bolus responses of the stresstransfer pathways characterized by second-order low-pass systems with constant kinetic rates but with different nonzero damping factors. The under-damped responses evaluated at $\alpha=\frac{1}{10} \omega_{0}$ has multiple periods of oscillations enveloped by an exponential decay function. The underdamped response evaluated at $\alpha=\frac{1}{2} \omega_{0}$ presents oscillatory pattern that quickly decays to zero. The over-damped response evaluated at $\alpha=10 \omega_{0}$ decays at a rate slower than the single first-order low-pass system shown in (A). The over-damped responses evaluated at $\alpha=2 \omega_{0}$ decays at a rate faster than the single first-order low-pass system and the dual in-parallel first-order low-pass system shown in (A). The critically damped response resembles the one of (A) that corresponds to the combination of 7 in-parallel first-order low-pass responses and resembles a hyperbolic pattern.

The (D) is specific to the bolus responses of the stresstransfer pathways characterized by a second-order bandpass systems with constant kinetic rates but with different nonzero damping factors. The under-damped response evaluated at $\alpha=\frac{1}{10} \omega_{0}$ has multiple periods of oscillations enveloped by an exponential decay function. The underdamped response evaluated at $\alpha=\frac{1}{2} \omega_{0}$ presented oscillatory pattern that quickly decays to zero. The over-damped responses evaluated at both $\alpha=10 \omega_{0}$ and $\alpha=2 \omega_{0}$ decay much faster than those in (C). The critically damped response also decays much faster than the one of $(A)$ that corresponds to the combination of 7 in-parallel first-order low-pass responses and is close to a hyperbolic pattern. The decay patterns over the first $1 \%$ of the duration are displayed in the inset figure. The over-damped response evaluated at $\alpha=10 \omega_{0}$ decays at a rate faster than $\alpha=2 \omega_{0}$, which is faster than the critically damped response.

\subsection{The responses of the hypothesized stress-transfer pathways to a step input}

The responses of the hypothesized model pathways of stress transfer to a step input are displayed in Fig. 5. The (A) specifies the step responses of the stress-transfer pathways characterized by single or multiple in-parallel firstorder low-pass systems having constant kinetic rates. The " $M$ " number indicates the number of first-order low-pass system hypothesized to be involved in the stress-transfer pathway. $M=1$ corresponds to a kinetic change defined by a single exponential function. $M=2$ corresponds to a kinetic change defined by a combination of two exponential functions. $M=7$ corresponds to a kinetic change defined by a combination of seven exponential functions. The kinetic rates of these exponential functions decrease exponentially according to the aforementioned principles. As the number of exponential functions increases, the uprising pattern deviates more and more from the singleexponential curve. The initial increase becomes faster and the saturating tail becomes longer. The transition between the faster initial increase and the slower later saturating tail is more pronounced when involving more first-order low-pass pathways.

The (B) is specific to the step responses of the stresstransfer pathways characterized by single first-order 
low-pass systems with a time-varying kinetic rate. The kinetic rate decreases exponentially according to the aforementioned principles. The step-response pattern for $K_{v}=0.00001$ is indistinguishable from the pattern of single first-order low-pass response defined by a fixed kinetic rate identical to the initial value of the kinetic rate of this system with a time-varying kinetics. As the kinetic rate varies faster, the step-response pattern deviates more and more from the single-exponential curve and starts to reveal biphasic pattern with a falling-phase slower than the rising phase. The initial uprising becomes faster that is followed by the peaking of the value and a later decay that grounds as the time increases. The falling phase becomes increasingly like a hyperbolic pattern as the rate of change of the kinetic constant increases. The biphasic pattern is also more pronounced as the rate of change of the kinetic constant increases.

The $(C)$ is specific to the step responses of the stresstransfer pathways characterized by second-order lowpass systems with constant kinetic rates but with different nonzero damping factors. The under-damped response evaluated at $\alpha=\frac{1}{10} \omega_{0}$ has multiple periods of oscillations enveloped by an exponential decay function to stabilize at a steady-state value of 1 . The under-damped response evaluated at $\alpha=\frac{1}{2} \omega_{0}$ presents oscillatory pattern that quickly stabilizes to a steady-state value of 1 . The overdamped response evaluated at $\alpha=10 \omega_{0}$ increases to the steady-state value of 1 at a rate slower than the single first-order low-pass system shown in (A). The over-damped responses evaluated at $\alpha=2 \omega_{0}$ increases to the steadystate value of 1 at a rate faster than the single first-order low-pass system and the dual in-parallel first-order lowpass system shown in (A). The critically damped response resembles the one of $(A)$ that corresponds to the combination of 7 in-parallel first-order low-pass responses.

The (D) is specific to the step responses of the stresstransfer pathways characterized by a second-order bandpass systems with constant kinetic rates but with different nonzero damping factors. The under-damped response evaluated at $\alpha=\frac{1}{10} \omega_{0}$ has multiple periods of oscillations enveloped by an exponential decay function that stabilizes to zero. The under-damped response evaluated at $\alpha=\frac{1}{2} \omega_{0}$ presented oscillatory pattern that quickly decays to zero. The over-damped responses evaluated at both $\alpha=10 \omega_{0}$ and $\alpha=2 \omega_{0}$ peak sharply then decay to zero, with the rate of the decay that is faster in $\alpha=2 \omega_{0}$ than in $\alpha=10 \omega_{0}$. The critically damped response decays after a sharp uprising, revealing a pattern similar to the one of $(A)$ of Fig. 4 that corresponds to the bolus response of the combination of 7 in-parallel first-order low-pass responses. The changes of the total five cases over the first $1 \%$ of the duration are displayed in the inset figure. The over-damped response evaluated at $\alpha=2 \omega_{0}$ increases slower than $\alpha=10 \omega_{0}$ but faster than the critically damped response.

\section{Discussion}

The commonly reported baseline spontaneous UPE is $100 \mathrm{~s}$ of photons per second per square centimeter [43-47]. At the visible wavelength of $500 \mathrm{~nm}$, a photon count rate of 1000 photons per second per square centimeter corresponds to an irradiance of $3.98 \times 10^{-16} \mathrm{~W} \cdot \mathrm{cm}^{-2}$ or $\sim 0.4 \mathrm{fW} \cdot \mathrm{cm}^{-2}$. This weak irradiance is comparable to the photon fluence rate measured at $10 \mathrm{~cm}$ from a source of $10 \mu \mathrm{W}$ power in an unbounded homogeneous tissue medium having an absorption coefficient of $0.106 \mathrm{~cm}^{-1}$ and a reduced scattering coefficient of $10 \mathrm{~cm}^{-1}$. However, when living organisms become stressed, stronger luminescence is observed [48]. The enhancement of photon counts above the baseline spontaneous UPE, i.e., the induced PE following exogenous stress renders the means of applying external stress-control to modulate the perturbation to homeostasis for probing the oxidative metabolism. However, major questions arise that include how to optimize (maximize) photon yield in association with a specific external stress, how to identify the number of metabolic pathways involved in the production of the photons emitted as induced PE which may also be insightful to the mechanism underlying baseline spontaneous UPE, and what physiological processes may be activated or involved for the metabolic pathways. These questions are difficult to address without identifying the kinetics of stress transfer, because all information of the metabolic response that will express photon counts in excess of the baseline spontaneous UPE can be exploited only by using the kinetics of induced PE, when the external stress can be modulated thus the stress-signal correlation could be optimized. More insights to the stress-transfer kinetics that sources the photo-genesis in excess of the baseline level for spontaneous UPE will thus help resolve how the stress type and pattern may be optimized to enhance the yield of induced PE for mechanistic discoveries and application developments.

\subsection{A hyperbolic stress-transfer kinetics may be the disguise of a multi-exponential stress transfer}

The kinetic patterns of induced PE do not appear to be simple. The hyperbolic decay pattern is the one that was 
resolved the most; however, the hyperbolically decaying pattern may be found from only approximately one-thirds of the reports. For the rest of the induced PE phenomena, there are more single-exponential cases than the patterns setting between the single-exponential and the hyperbolic ones. The patterns that set between the single-exponential and the hyperbolic ones are accountable by using two or more exponential functions. Note that the hyperbolic function fitting the kinetics of induced PE appears as a compressed form, and a compressed hyperbolic function can in principle be decomposed to the summation of an infinite number of exponential functions [42] through a Laplace transform approach. In such decomposition, the coefficient of each exponential function with negative argument corresponds to a probability distribution of the kinetic rate of the negative argument. Therefore, a hyperbolic function is essentially a combination of many exponential functions of which the one with longer time constant has lower amplitude thus matters less and weights in later. Since each exponential component of negative argument constitutes a first-order low-pass response, a lesser amount of exponential functions contributing to the decay kinetics will make the kinetic decay pattern look more like a pure exponential decay. Conversely, the involvement of more individually pure exponential kinetics can make the composite kinetic pattern deviate more from a pure exponential pattern and look more like a hyperbolic pattern. That is the case shown in Fig. 4a for the response to a bolus input by the stress-transfer pathways hypothesized to consist of one, two, and seven exponential functions. Therefore, frequency-domain analysis may be needed to determine the true kinetic components of a hyperbolically decaying induced PE.

\subsection{A hyperbolic pattern may be the indication of time-varying kinetics of a simple stress transfer}

What are perhaps more informative and interesting are the bolus and step responses of the hypothesized stresstransfer pathway of a first-order low-pass behavior but operated with a varying kinetic rate that decreases over time or equivalently the time constant increases over time as shown in Figs. $4 \mathrm{~b}$ and $5 \mathrm{~b}$. The response of this system shall approach that of the single and pure exponential function when the kinetic rate barely changes over the duration of the acquisition. As the change of the kinetic rate increases, the response to both the bolus input and the step input becomes more and more like a hyperbolic pattern that starts from an initial peak in association with a bolus input or appears after a rapidly occurring peak in association with a step input. Such pattern of change was found in the oxidation current of superoxide anion radical
$\left(\mathrm{O}_{2}^{--}\right)$in response to wound-stress as was investigated electrochemically [49]. Such behavior can be appreciated from the perspective of the frequency spectrum of the impulse response of the system of the stress transfer. The kinetic rate of a first-order exponential function with the argument decaying over time is the low-pass cutoff frequency of the frequency spectrum of the impulse response. A faster kinetic rate means a higher cutoff frequency of the low-pass filtering, so an increased kinetic rate corresponds to increased passage of high-frequency components. The rising phase of the step stress carries the same frequency components as the bolus input, whereas the steady-state phase of the step stress gives the zero-frequency component. As the low-pass cutoff frequency is increased, more high-frequency components of the input will be passed so the output will resemble more of the response to a bolus input that has charged the system with an initial energy. Therefore, the response to a step stress may be visually more informative than the response to a simple bolus input, in identifying stress-transfer responses that cannot be described by a simple exponential decay with a fixed kinetic rate. Similar kinetic behavior can also be found with the over-damped case of the second-order band-pass responses. The over-damped case with secondorder band-pass response is associated with two exponential functions having negative arguments. The kinetic rate of the two exponential functions that is greater thus sets the low-pass cutoff frequency of the band-pass behavior, and so a larger kinetic rate will pass more high-frequency components of the input to make the response to a step input closer to that of the over-damped response to a bolus input. This again indicated the need of frequencydomain analysis to determine the true kinetic components of a hyperbolically decaying induced PE. In fact, the use of frequency components of the induced PE to inform metabolic process has been indicated [50]. More robust frequency-spectrum analysis of the induced PE can be expected if the induced PE can be acquired at a signalto-noise ratio much stronger than is available now, and that would require modeling of the kinetics to facilitate instrumentation approaches that would enhance the yield of induced PE.

\subsection{What is special about the stress-transfer kinetics corresponding to dual-exponential decay?}

It is noted that, the decomposition of a hyperbolic function to exponential functions is mechanistically similar to Fourier series expansion of a periodical function. It is thus straightforward to stipulate that the most dominant kinetic component of the decomposition of the hyperbolic function will be the one that varies the fastest thus has the 
highest kinetic weight with it, just like the fundamentalfrequency component of a Fourier series expansion. And the kinetic component that varies slower should have a smaller kinetic weight with it. That is the principle used to set the initial values of the individual single-exponential components of the stress-transfer pathway, where an exponential function with a slower (smaller) kinetic rate is assigned with a smaller initial value of that exponential function. In this sense, the kinetic pattern of the dualexponential function may need some attentions. According to Eq. (20), the two exponential components have the ratio between their initial values identical to the ratio between their kinetic rates. If a kinetic decay of monotonic change does not fit to a single exponential function, the next easiest fit will be a dual-exponential pattern that will produce two numbers for the kinetic rate or the inverse of the time constant, and two numbers for the initial value or the intensity of each exponential component. So if a kinetic decay pattern can be fitted with two exponential functions, one can identify if the kinetic pathway may be considered as consisting of two parallel first-order lowpass responses, by comparing the ratio of the fitted intensities of the two exponential functions and the ratio of the kinetic rates of the two functions.

The correlation between the intensity ratio and kineticrate ratio of a decay pattern when it can be fitted with dual-exponential functions was actually inferred [51] in relating to the ways of Nitellopsis obtusa cells exposed to ascorbic acid (AsA) at concentrations regarded inadequate to penetrate into the cell. The time-dependence of induced PE in excess of the baseline UPE from isolated cell membranes or walls stressed by AsA expressed a single kinetic rate in the decaying phase. In comparison, the time-dependence of induced PE in excess of the baseline UPE from intact cells treated with high concentrations of AsA expressed a kinetic pattern expressing a doubleexponential pattern with the slower phase comparable to the response of cell-wall alone to the stress by AsA in terms of the kinetic rate. It was speculated based on a physically simple model of the cell that, a small portion of AsA (lower concentrations) insufficient to penetrate into the cell would cause the biochemical reactions responsible for induced PE to take place mainly within the cell walls. In comparison, a large portion of AsA (higher concentrations) acting on whole cell allowed the reagent to penetrate the whole cell to cause the biochemical reactions responsible for induced PE to take place in the cytoplasm, in addition to that taking place in the wall. Therefore, the stress by AsA strong enough to act on the cytoplasm through wall could cause a more direct (thus stronger and faster) stress-transfer process between the stress application and the alteration of photo-genesis through oxidative reduction metabolism, besides the slower path via the cell wall. It was also suggested that induced PE from the intact cells would not be a simple sum of emission from separate subcellular fractions due to the reabsorption and re-emission processes expected in the whole intact cells. That notion helps interpret that the cytoplasm exposed to the AsA expressed a double-exponential kinetic pattern in the decaying phase of the induced PE very close to that expressed by the intact cell exposed to higher concentration of AsA. For the temporal patterns expressed by the cytoplasm alone after exposing to AsA, the ratio of the kinetic rates of the double-exponential fit was 8.5, which is noticeably close ( $3 \%$ different) to the ratio of 8.23 between the amplitudes of the respective exponential functions. The closeness of the two ratios implies possible involvement of two globally parallel first-order stress-transfer processes responsible for induced PE when the cytoplasm was stressed by AsA. To investigate which pathways or sources of induced PE could play a role during AsA stressing of the cytoplasm alone, the spectral variation of the induced PE with respect to the baseline spontaneous UPE may need be analyzed in conjunction with the temporal information and that would require spectrally and temporally coupled analysis of the transfer-kinetics.

\subsection{Faster stress-transfer kinetics may correlate with stronger initial induction and it has implication to enhancing the yield of induced PE}

The bolus response of a single exponential system, or the one with the largest kinetic rate of a multiple in-parallel low-pass system deserves attention. It should be noted that the response to a bolus input also represents the response to the falling phase when a step input is stopped, and that should indicate what may be expected following the falling edge of a pulse stress. Equation (20) for the response of a single first-order low-pass system to a bolus input implies that, a greater kinetic rate of photo-genesis by the metabolic engine will not only cause the decay to be faster, but also make the initial intensity to be stronger, at the condition of the same kinetic rate preceding the metabolic engine. This may be especially insightful to why the induced PE with shorter delay time or faster decay rate is generally stronger than those having longer delay time or slower decay rate.

There is a quite significant discrepancy of the kinetic rate of induced PE between that caused by non-photic stress and that by photic stress. It may worth to speculate how this discrepancy could indicate the temporal difference in terms of stress-transfer process between non-photic stress and photic stress. The energy for weak photon emission is produced when an excited biological molecule like ROS drops to a lower-energy state. The gap 
of the energy states between which the excited biological molecule makes the transition characterizes the spectrum of the photon emission, whereas the rate of transition determines the intensity of the photon emission. A baseline spontaneous UPE revealing a steady-state spectral intensity pattern corresponds to a steady-state rate of transition of ROS, which further indicates a dynamic balance between the production and scavenge of ROS. When stressed, the photon emission in excess of the baseline spontaneous UPE comes from increased rate of transition of ROS, which may be caused by increased production, decreased scavenging, or shortened lifetime of ROS in the excited state. Regardless of how the photo-genesis (transition between energy states) occurs, an external stress must be picked up by a receptor than passed and processed along the receptor-metabolic chain to the terminal process to increase the rate of transition of ROS to cause an elevation of photon emission in excess of the baseline spontaneous UPE. A photic stress may be able to directly affect photo-sensitive constitutes in the metabolic pathway responsible for promoting total rate of ROS transition. Whereas a non-photic stress would require additional intermediate states that are either local or systematic, that will slow their effects down in terms of triggering the same type of responses in the terminal metabolic engine process of photo-genesis in comparison with what the photic stress would be able to activate. Stress transfer in response to thermal-stress, for example, would be slower than stress-transfer responding to photic stress, because high temperature will need to activate thermo-receptors to inactivate antioxidant enzymes and other enzymes to promote lipid peroxidation by ROS that are not detoxified by the enzymes [1].

The phenomenon of stronger initial intensity associated with faster decay kinetics renders the potential to enhance the yield of induced PE by reducing the time lag between the removal of the stress and the starting of the acquisition of induced PE if the stress can be turned off cleanly (i.e., no after-glow for photic sources). The shortest device-specific time-delay that has been used for induced PE acquisition is $8.5 \mu \mathrm{s}$, and the kinetic pattern associated with photicstressed induced PE generally reveal a one order of magnitude change of intensity over one order of magnitude change of time at the initial collection stage. Thus, photon counting started at one-order of magnitude of time faster, i.e., $0.85 \mu \mathrm{s}$, may be accompanied with the initial photon count of one order of magnitude greater than what has been reported for photic stress. Should the temporal delay between shutting off the photic stress and starting the photon acquisition be further reduced, the initial photoncount of the induced PE may be further increased. It has been shown with the existing reports that the initial photon count of the induced PE in response to photic stress can be as high as 5 orders of magnitude greater than the baseline spontaneous UPE [11], but remains 5-6 orders of magnitude weaker than the weak fluorescence signals excited by Cerenkov illumination [52]. It is thus possible to have a photon-count level of induced PE to reach the level of Cerenkov excited fluorescence, should the induced PE be acquired at a timescale at least 2 orders of magnitude faster than what has been accomplished now. This projection will certainly be incorrect, should the kinetics of the pre-8.5 $\mu$ s duration of photic-stressed induced PE not follow the same first-order kinetics as appearing initially after the $8.5 \mu \mathrm{s}$ of starting the acquisition. If that were the case, the initial stress-transfer response would not be characterized by a first-order low-pass system having a fixed kinetic rate, but rather it may indicate either a first-order system with a varying kinetic rate or a second-order system that is over-damped. Faster time-correlated photon-counting to reveal the initial temporal pattern of induced PE following a stress will thus help resolve if the stress transfer follows a simple first-order low-pass mechanism with a fixed or varying metabolic rate or shall be considered a secondorder process involving negative feedbacks at two stages.

\subsection{Limitations of the approaches}

Many assumptions implemented in this system-based modeling work regarding the kinetic rates of the stresstransfer pathway may seem overly simple and arbitrary. For example, the kinetic rate channeling the stress to the summating engine referring to a metabolic mechanism of photo-genesis is a constant. With this assumption of a constant rate for feeding the metabolic engine of photogenesis, the intensity of source production as the output of the stress-transfer pathway will be proportional to the input, i.e., linearly dependent on the stress intensity. That does not agree with many patterns of induced PE in response to particularly photic stress. It is however noted that biological responses to external perturbations have limits. When the external perturbation exceeds a threshold of the biological response, the integrity of the physical or functional pathways may be altered such that the response is no longer a quantitative change of the same proportion but rather a reduced or saturated response so the model applying to a quantitative variation of an integral entity of the same physical and functional manifestations will fail. The biological response of this kind of system to the input can be expected to be nonlinearly dependent upon the intensity of the input, causing the kinetic rate to saturate as the intensity of the stress approaches a threshold and that will cause the photo-genesis to saturate as the intensity approaches the threshold. The saturation of the kinetic rate in stress transfer affecting the metabolic engine of photo-genesis could serve as an alternative to 
the multi-soliton coherent-state perspective $[18,19,36$, 37] that has helped interpret the nonlinear dependence of the photon count in induced PE as a function of the stress intensity.

Many other aspects of this work could be augmented. For example, the fast-oscillatory response overlapping on a slowly changing global profile that deviates from the baseline level may invite the combination of a secondorder under-damped pathway with a first-order low-pass pathway or another second-order pathway that is overdamped. The kinetic rate of induced PE responding to a rising phase of a stress may also differ from that to a falling phase of the stress even for a pulsed stress, since one of the rising and falling phases may trigger an agonistic or stimulatory pathway and the other may relate to an antagonistic or inhibitory pathway. Should that be the case then the level of the intensity change of induced PE would differ between the rising phase and the falling phase of the same pulse stress [23]. That difference would nevertheless be proportional to the kinetic rate of the initial change that can be fitted with exponential decaying functions. In terms of the response to a stress that repeats, the stresstransferring output can be readily identified by comparing the scales of the period of the repeating stress and the responding time of the pathway. For the cases of the pathway exhibiting first-order low-pass response, the longest time constant will determine how the cumulative response will change. Apparently, if the period of the repeating stress is much longer (e.g., $>5$ times) than the longest time constant of the stress-transfer pathway, the response to the repetitive stress will be a simple repetition of the response to one period of the repeating stress. If however the period of the repeating stress is comparable to the longest time constant of the stress-transfer pathway, the response to the repetitive stress will have the response of the pathway to a single period of the input added to that to a previous period that has not stabilized completely, and that will cause the upper and lower boundaries of the responses to change nonlinearly, likely to manifest a slowly exponentially increasing pattern [23]. If the period of the repeating stress, or the duration of a step stress is even smaller than the shortest time constant of the stresstransfer pathway, the response to the repeating stress or a step stress may manifest either a near-linear change of the photon count similar to heat shock-induced PE [1] or linear increment of the photon count as the stress repeats [23]. Further modulation of the stress patterns (such as applying the stress in a chirped way) may allow coherent detection techniques be applied to significantly enhance the signal-to-noise ratio (SNR) of acquiring induced PE for faster throughput that will benefit the probing of specific oxidative metabolism for broader applications.

\section{Conclusion}

This work has speculated that induced PE involves a stresstransfer pathway that kinetically produces the surfaceemitted photons at a temporal rate much slower than the lifetime of photon propagation in tissue. Modeling the kinetics of stress-transfer pathway with a linear-system approach illustrates how a single stress-transfer pathway may manifest various photo-genesis patterns in responding to the same stress-input, and why a single kinetic pattern of photo-genesis may arise from multiple paths of stress transfer. It is shown that the hyperbolic decay pattern (common to induced PE) of photo-genesis can be resulted from a number of pathway configurations, including multiple in-parallel first-order low-pass pathways each having fixed kinetic rate when responding to a bolus input, and a single low-pass pathway having a gradually decreasing kinetic rate when responding to a bolus or a step input. The pattern of photo-genesis from a pathway containing two parallel first-order low-pass transfer mechanism is uniquely informative in terms of how the kinetics rates and the intensities of the two phases shall relate. $A$ stress transfer with a first-order low-pass mechanism having a slowly reducing kinetic rate can produce patterns of photo-genesis that are disguisable of the responses to a few other stress-transfer mechanisms. The patterns of photo-genesis from a pathway of second-order characteristics are unique when the kinetic rates allow damped oscillatory response to develop. These indications may be considered for instrumenting methods to enhance the yield based on induced PE.

\section{Compliance with ethical standards}

Conflict of interest The author declares that he has no conflict of interest.

\section{References}

1. Kobayashi K, Okabe H, Kawano S, Hidaka Y, Hara K (2014) Biophoton emission induced by heat shock. PLoS ONE 9(8):e105700

2. Tsuchida K, Iwasa T, Kobayashi M (2019) Imaging of ultraweak photon emission for evaluating the oxidative stress of human skin. J Photochem Photobiol B 198:111562

3. Slawinski J, Ezzahir A, Godlewski M, Kwiecinska T, Rajfur Z, Sitko D, Wierzuchowska D (1992) Stress-induced photon emission from perturbed organisms. Experientia 48(11-12):1041-1058

4. Musumeci F, Scordino A, Triglia A (1997) Delayed luminescence from simple biological systems. Riv Biol 90(1):95-110

5. Burgos RCR, Zhang W, van Wijk EPA, Hankemeier T, Ramautar $\mathrm{R}$, van der Greef J (2017) Cellular glutathione levels in HL-60 cells during respiratory burst are not correlated with ultra-weak photon emission. J Photochem Photobiol B 175:291-296 
6. Burgos RCR, Schoeman JC, Winden LJV, Cervinkova K, Ramautar R, Van Wijk EPA, Cifra M, Berger R, Hankemeier T, Greef JV (2017) Ultra-weak photon emission as a dynamic tool for monitoring oxidative stress metabolism. Sci Rep 7(1):1229

7. Rac M, Sedlarova M, Pospisil P (2015) The formation of electronically excited species in the human multiple myeloma cell suspension. Sci Rep 5:8882

8. Scordino A, Campisi A, Grasso R, Bonfanti R, Gulino M, lauk L, Parenti R, Musumeci F (2014) Delayed luminescence to monitor programmed cell death induced by berberine on thyroid cancer cells. J Biomed Opt 19(11):117005

9. Jain A, Rieger I, Rohr M, Schrader A (2010) Antioxidant efficacy on human skin in vivo investigated by UVA-induced chemiluminescence decay analysis via induced chemiluminescence of human skin. Skin Pharmacol Physiol 23(5):266-272

10. Niggli HJ, Tudisco S, Privitera G, Applegate LA, Scordino A, Musumeci $F$ (2005) Laser-ultraviolet-A-induced ultraweak photon emission in mammalian cells. J Biomed Opt 10(2):024006

11. Baran I, Ionescu D, Privitera S, Scordino A, Mocanu MM, Musumeci F, Grasso R, Gulino M, Iftime A, Tofolean IT, Garaiman A, Goicea A, Irimia R, Dimancea A, Ganea C (2013) Mitochondrial respiratory complex I probed by delayed luminescence spectroscopy. J Biomed Opt 18(12):127006

12. Oros CL, Alves $F(2018$ ) Leaf wound induced ultraweak photon emission is suppressed under anoxic stress: observations of Spathiphyllum under aerobic and anaerobic conditions using novel in vivo methodology. PLoS ONE 13(6):e0198962

13. Goraczko W, Slawinski J (2004) Secondary ultraweak luminescence from humic acids induced by gamma-radiation. Nonlinearity Biol Toxicol Med 2(3):245-258

14. Maccarrone M, Fantini C, Agro AF, Rosato N (1998) Kinetics of ultraweak light emission from human erythroleukemia K562 cells upon electroporation. Biochim Biophys Acta 1414(1-2):43-50

15. Červinková, M.B.L.J.M.C.K (2016) Low frequency electromagnetic field effects on ultra-weak photon emission from yeast cells. In: 2016 ELEKTRO

16. Volodyaev I, Beloussov LV (2015) Revisiting the mitogenetic effect of ultra-weak photon emission. Front Physiol 6:241

17. Alvermann M, Srivastava YN, Swain J, Widom A (2015) Biological electric fields and rate equations for biophotons. Eur Biophy J Biophys Lett 44(3):165-170

18. Scordino A, Grasso R, Gulino M, Lanzano L, Musumeci F, Privitera G, Tedesco M, Triglia A, Brizhik L (2010) Delayed luminescence from collagen as arising from Soliton and small Polaron states. Int J Quantum Chem 110(1):221-229

19. Popp FA, Li KH (1993) Hyperbolic relaxation as a sufficient condition of a fully coherent ergodic field. Int J Theor Phys 32(9):1573-1583

20. Kataoka Y, Cui YL, Yamagata A, Niigaki M, Hirohata T, Oishi N, Watanabe $Y$ (2001) Activity-dependent neural tissue oxidation emits intrinsic ultraweak photons. Biochem Biophys Res Commun 285(4):1007-1011

21. Sauermann G, Mei WP, Hoppe U, Stab F (1999) Ultraweak photon emission of human skin in vivo: influence of topically applied antioxidants on human skin. Methods Enzymol 300:419-428

22. Inaba $\mathrm{H}$ (1988) Super-high sensitivity systems for detection and spectral analysis of ultraweak photon emission from biological cells and tissues. Experientia 44(7):550-559

23. Footitt $\mathrm{S}$, Palleschi S, Fazio E, Palomba R, Finch-Savage WE, Silvestroni $L$ (2016) Ultraweak photon emission from the seed coat in response to temperature and humidity-a potential mechanism for environmental signal transduction in the soil seed bank. Photochem Photobiol 92(5):678-687

24. Betogna EG, et al. (2016) Design and implementation of a measurement system for ultra-weak bioluminescence detection from
E. coli cultures applied to sanitary control. In: 2016 12th IEEE International conference on industry applications (INDUSCON), Curitiba, 2016, pp 1-4

25. Winkler R, Guttenberger H, Klima H (2009) Ultraweak and induced photon emission after wounding of plants. Photochem Photobiol 85(4):962-965

26. Bajpai RP, Bajpai PK, Roy D (1991) Ultraweak photon emission in germinating seeds: a signal of biological order. J Biolumin Chemilumin 6(4):227-230

27. Shanei A, Alinasab Z, Kiani A, Nematollahi MA (2017) Detection of ultraweak photon emission (UPE) from cells as a tool for pathological studies. J Biomed Phys Eng 7(4):389-396

28. Hossu M, Ma L, Chen W (2010) Nonlinear enhancement of spontaneous biophoton emission of sweet potato by silver nanoparticles. J Photochem Photobiol B 99(1):44-48

29. Kageyama C, Kato K, lyozumi H, Inagaki H, Yamaguchi A, Furuse K, Baba K (2006) Photon emissions from rice cells elicited by $\mathrm{N}$-acetylchitooligosaccharide are generated through phospholipid signaling in close association with the production of reactive oxygen species. Plant Physiol Biochem 44(11-12):901-909

30. Slawinska D, Slawinski J (1997) Chemiluminescence of cereal products. I. Kinetics, activation energy and effects of solvents. J Biolumin Chemilumin 12(5):249-259

31. Kimura M, Roschger P, Kobayashi M, Kimura S, Inaba H (1992) $\mathrm{N}$-methyl- $\mathrm{N}^{\prime}$-nitro- $\mathrm{N}$-nitrosoguanidine-induced light emission in Chinese hamster cell cultures: correlation with enhancement of chromosomal aberrations. Mutat Res 281(3):215-220

32. Slawinska D, Slawinski J (1987) Ultraweak photon emission in model reactions of the in vitro formation of eumelanins and pheomelanins. Pigment Cell Res 1(3):171-175

33. Barsacchi R, Camici P, Bottigli U, Salvadori PA, Pelosi G, Maiorino M, Ursini F (1983) Correlation between hydroperoxide-induced chemiluminescence of the heart and its function. Biochim Biophys Acta 762(2):241-247

34. Brizhik L, Musumeci F, Scordino A, Triglia A (2000) The soliton mechanism of the delayed luminescence of biological systems. Europhys Lett 52(2):238-244

35. Popp FA, Yan Y (2002) Delayed luminescence of biological systems in terms of coherent states. Phys Lett A 293(1-2):93-97

36. Brizhik L, Musumeci F, Scordino A, Tedesco M, Triglia A (2003) Nonlinear dependence of the delayed luminescence yield on the intensity of irradiation in the framework of a correlated soliton model. Phys Rev E 67(2):021902

37. Brizhik L, Scordino A, Triglia A, Musumeci F (2001) Delayed luminescence of biological systems arising from correlated manysoliton states. Phys Rev E 64(3):031902

38. Chwirot WB (1988) Ultraweak photon emission and anther meiotic cycle in Larix europaea (experimental investigation of $\mathrm{Nagl}$ and Popp's electromagnetic model of differentiation). Experientia 44(7):594-599

39. Piao D (2020) On the delayed ultraweak photon emission from organism in response to stress: I, how much temporal delay can be attributed to the tissue scattering of light?. SN Appl Sci. (Submitted)

40. Rivlin-Etzion M, Marmor O, Saban G, Rosin B, Haber SN, Vaadia E, Prut Y, Bergman H (2008) Low-pass filter properties of basal ganglia cortical muscle loops in the normal and MPTP primate model of parkinsonism. J Neurosci 28(3):633-649

41. Wlodarczyk J, Kierdaszuk B (2003) Interpretation of fluorescence decays using a power-like model. Biophys J 85(1):589-598

42. Whitehead L, Whitehead R, Valeur B, Berberan-Santos M (2009) A simple function for the description of near-exponential decays: the stretched or compressed hyperbola. Am J Phys 77(2):173-179

43. Cohen S, Popp FA (1997) Biophoton emission of the human body. J Photochem Photobiol B 40(2):187-189 
44. Nakamura K, Hiramatsu M (2005) Ultra-weak photon emission from human hand: influence of temperature and oxygen concentration on emission. J Photochem Photobiol B 80(2):156-160

45. Jung HH, Yang JM, Woo WM, Choi C, Yang JS, Soh KS (2005) Yearlong biophoton measurements: normalized frequency count analysis and seasonal dependency. J Photochem Photobiol B 78(2):149-154

46. Ou-Yang $\mathrm{H}$ (2014) The application of ultra-weak photon emission in dermatology. J Photochem Photobiol B 139:63-70

47. Zhao $X$, van Wijk E, Yan $Y$, van Wijk $R$, Yang $H$, Zhang $Y$, Wang J (2016) Ultra-weak photon emission of hands in aging prediction. J Photochem Photobiol B 162:529-534

48. Cadenas E, Arad ID, Boveris A, Fisher AB, Chance B (1980) Partial spectral analysis of the hydroperoxide-induced chemiluminescence of the perfused lung. FEBS Lett 111(2):413-418

49. Prasad A, Kumar A, Matsuoka R, Takahashi A, Fujii R, Sugiura Y, Kikuchi H, Aoyagi S, Aikawa T, Kondo T, Yuasa M, Pospisil P, Kasai S (2017) Real-time monitoring of superoxide anion radical generation in response to wounding: electrochemical study. PeerJ 5:e3050

50. Beloussov LVea (2003) Statistical and frequency-amplitude characteristics of ultraweak emissions of the Loach eggs and embryos under the normal conditions and upon their optical interactions. 2. Change in characteristics of ultraweak emissions upon optic interactions of groups of embryos of different ages. Russ J Develop Biol 34(6):379-388

51. Jaskowska A, Borc R, Milczarek I, Dudziak A, Spiewla E (2001) Kinetics studies of ultraweak luminescence induced by ascorbic acid in Characeae cells and their structures. Luminescence 16(1):51-56

52. Tanha K, Pashazadeh AM, Pogue BW (2015) Review of biomedical Cerenkov luminescence imaging applications. Biomed Opt Express 6(8):3053-3065

Publisher's Note Springer Nature remains neutral with regard to jurisdictional claims in published maps and institutional affiliations. 